Supporting Informations

Insertion of $\mathrm{CO}$ and Alkenes into the (Methyl)Pd Complexes of the Bidentate Porphyrin Ligand.

Jun-ichiro Setsune,* Takashi Yamauchi, Sachiko Tanikawa, Yuka Hirose, and Junya Watanabe

Department of Chemistry, Faculty of Science, and Graduate School of Science and Technology, Kobe University, Nada-ku, Kobe 657-8501

Table 1. Crystal data and structure refinement for (4). P2

Table 2. Atomic coordinates $\left(\times 10^{4}\right)$ and equivalent isotropic displacement parameters $\left(\AA^{2} \times 10^{3}\right)$ for (4). P2

Table 3. Bond lengths $[\AA]$ and angles $\left[{ }^{\circ}\right]$ for (4). P5

Table 4. Anisotropic displacement parameters $\left(\AA^{2} \times 10^{3}\right)$ for (4). P11

Table 5. Hydrogen coordinates $\left(x 10^{4}\right)$ and isotropic displacement parameters $\left(\AA^{2} \mathrm{x} 10^{3}\right)$ for (4). P14

Table 6. Least-squares planes (x,y,z in crystal coordinates) and deviations from them for (4). P15

Table 7. Crystal data and structure refinement for (5). P17

Table 8. Atomic coordinates $\left(x 10^{4}\right)$ and equivalent isotropic displacement parameters $\left(\AA^{2} \times 10^{3}\right)$ for $(\mathbf{5})$. P19

Table 9. Bond lengths $[\AA]$ and angles $\left[^{\circ}\right]$ for (5). P20

Table 10. Anisotropic displacement parameters $\left(\AA^{2} \times 10^{3}\right)$ for (5). P29

Table 11. Hydrogen coordinates $\left(x 10^{4}\right)$ and isotropic displacement parameters $\left(\AA^{2} \times 10^{3}\right)$ for $(\mathbf{5})$. P31

Table 12. Least-squares planes ( $x, y, z$ in crystal coordinates) and deviations from them for (5). P33

Figure 1. Ortep drawing with atom numbering scheme (50\% level thermal ellipsoids) (4). P37

Figure 2. Ortep drawing with atom numbering scheme (50\% level thermal ellipsoids) (5). P38 
Table 1. Crystal data and structure refinement for (4).

\begin{tabular}{|c|c|c|}
\hline Identification code & \multicolumn{2}{|c|}{ (por) $\mathrm{Pd}(\mathrm{C}(\mathrm{O}) \mathrm{Me})(\mathrm{Cl}) \cdot \mathrm{CH}_{2} \mathrm{Cl}_{2} \cdot \mathrm{H}_{2} \mathrm{O}$} \\
\hline Empirical formula & \multicolumn{2}{|c|}{ C61 H45 Cl3 N4 O2 Pd } \\
\hline Formula weight & \multicolumn{2}{|l|}{1078.76} \\
\hline Temperature & \multicolumn{2}{|l|}{ 293(2) K } \\
\hline Wavelength & \multicolumn{2}{|l|}{$0.71073 \AA$} \\
\hline Crystal system & \multicolumn{2}{|l|}{ monoclinic } \\
\hline Space group & \multicolumn{2}{|l|}{$\mathrm{P} 2(1) / \mathrm{c}$} \\
\hline \multirow[t]{3}{*}{ Unit cell dimensions } & $\mathrm{a}=11.8443(10) \AA$ & $\mathrm{a}=90^{\circ}$ \\
\hline & $\mathrm{b}=18.6502(15) \AA$ & $\mathrm{b}=103.482(2)^{\circ}$ \\
\hline & $\mathrm{c}=24.072(2) \AA$ & $\mathrm{g}=90^{\circ}$ \\
\hline Volume & \multicolumn{2}{|l|}{$5170.9(7) \AA^{3}$} \\
\hline $\mathrm{Z}$ & \multicolumn{2}{|l|}{4} \\
\hline Density (calculated) & \multicolumn{2}{|l|}{$1.386 \mathrm{Mg} / \mathrm{m}^{3}$} \\
\hline Absorption coefficient & \multicolumn{2}{|l|}{$0.562 \mathrm{~mm}^{-1}$} \\
\hline $\mathrm{F}(000)$ & \multicolumn{2}{|l|}{2208} \\
\hline Crystal size & \multicolumn{2}{|l|}{$0.3 \times 0.2 \times 0.1 \mathrm{~mm}^{3}$} \\
\hline Theta range for data collection & \multicolumn{2}{|l|}{1.40 to $27.23^{\circ}$} \\
\hline Index ranges & \multicolumn{2}{|c|}{$-15<=\mathrm{h}<=14,-10<=\mathrm{k}<=22,-29<=\mathrm{l}<=28$} \\
\hline Reflections collected & \multicolumn{2}{|l|}{27688} \\
\hline Independent reflections & \multicolumn{2}{|c|}{$10269[\mathrm{R}(\mathrm{int})=0.0520]$} \\
\hline Completeness to theta $=$ & \multicolumn{2}{|l|}{$27.23^{\circ} \quad 88.9 \%$} \\
\hline Refinement method & \multicolumn{2}{|c|}{ Full-matrix least-squares on $\mathrm{F}^{2}$} \\
\hline Data / restraints / parameters & \multicolumn{2}{|l|}{10269 / 0 / 648} \\
\hline Goodness-of-fit on $\mathrm{F}^{2}$ & \multicolumn{2}{|l|}{1.003} \\
\hline Final R indices [I>2sigma(I)] & \multicolumn{2}{|c|}{$\mathrm{R} 1=0.0576, w \mathrm{R} 2=0.1571$} \\
\hline $\mathrm{R}$ indices (all data) & \multicolumn{2}{|c|}{$\mathrm{R} 1=0.0967, \mathrm{wR} 2=0.1812$} \\
\hline Largest diff. peak and hole & \multicolumn{2}{|c|}{1.993 and -0.693 e. $\AA^{-3}$} \\
\hline
\end{tabular}


Table 2. Atomic coordinates ( $\left.\times 10^{4}\right)$ and equivalent isotropic displacement parameters $\left(\AA^{2} \times 10^{3}\right)$ for $(4)$. U(eq) is defined as one third of the trace of the orthogonalized Uij tensor.

\begin{tabular}{|c|c|c|c|c|}
\hline & $\mathrm{X}$ & $\mathrm{y}$ & $\mathrm{z}$ & $\mathrm{U}(\mathrm{eq})$ \\
\hline $\mathrm{C}(1)$ & $2069(4)$ & $753(2)$ & $8361(2)$ & $33(1)$ \\
\hline$C(2)$ & $1247(4)$ & $862(3)$ & $8698(2)$ & $43(1)$ \\
\hline$C(3)$ & $1783(4)$ & 1184(3) & $9194(2)$ & $42(1)$ \\
\hline $\mathrm{C}(4)$ & $2964(4)$ & $1289(2)$ & $9192(2)$ & $36(1)$ \\
\hline$C(5)$ & $3763(4)$ & 1713(3) & $9568(2)$ & $37(1)$ \\
\hline$C(6)$ & $4790(4)$ & 1973(2) & $9448(2)$ & $37(1)$ \\
\hline$C(7)$ & $5469(4)$ & $2547(3)$ & $9727(2)$ & $48(1)$ \\
\hline$C(8)$ & $6307(4)$ & $2685(3)$ & $9442(2)$ & $49(1)$ \\
\hline $\mathrm{C}(9)$ & $6199(4)$ & $2210(3)$ & $8982(2)$ & $36(1)$ \\
\hline $\mathrm{C}(10)$ & $6907(4)$ & $2229(2)$ & $8588(2)$ & $36(1)$ \\
\hline $\mathrm{C}(11)$ & $6659(4)$ & $1922(2)$ & $8045(2)$ & $36(1)$ \\
\hline $\mathrm{C}(12)$ & $7529(4)$ & $1785(3)$ & $7722(2)$ & $44(1)$ \\
\hline$C(13)$ & $6999(4)$ & $1435(3)$ & $7248(2)$ & $46(1)$ \\
\hline $\mathrm{C}(14)$ & $5802(4)$ & $1349(2)$ & $7265(2)$ & $35(1)$ \\
\hline$C(15)$ & $5003(4)$ & $932(2)$ & $6883(2)$ & $36(1)$ \\
\hline$C(16)$ & $3908(4)$ & $722(3)$ & $6969(2)$ & $39(1)$ \\
\hline$C(17)$ & $3152(4)$ & 198(3) & $6632(2)$ & $49(1)$ \\
\hline $\mathrm{C}(18)$ & $2280(4)$ & $83(3)$ & $6887(2)$ & $49(1)$ \\
\hline C(19) & $2486(4)$ & $521(3)$ & $7395(2)$ & $38(1)$ \\
\hline $\mathrm{C}(20)$ & $1803(4)$ & $482(2)$ & $7803(2)$ & $37(1)$ \\
\hline $\mathrm{C}(21)$ & $3407(4)$ & 2011(3) & $10085(2)$ & $40(1)$ \\
\hline $\mathrm{C}(22)$ & $3407(5)$ & 1592(4) & $10553(2)$ & $60(2)$ \\
\hline $\mathrm{C}(23)$ & $3100(6)$ & $1894(5)$ & $11029(3)$ & $83(2)$ \\
\hline $\mathrm{C}(24)$ & $2803(6)$ & $2613(5)$ & $11030(3)$ & $80(2)$ \\
\hline$C(25)$ & $2797(6)$ & $3014(4)$ & $10568(3)$ & $77(2)$ \\
\hline
\end{tabular}




\begin{tabular}{|c|c|c|c|c|}
\hline$C(26)$ & $3088(5)$ & $2719(3)$ & $10088(3)$ & $56(1)$ \\
\hline$C(27)$ & $8040(4)$ & $2600(3)$ & $8770(2)$ & $39(1)$ \\
\hline $\mathrm{C}(28)$ & $8832(5)$ & 2394(3) & $9275(2)$ & $54(1)$ \\
\hline$C(29)$ & $9903(5)$ & $2725(4)$ & $9429(3)$ & $66(2)$ \\
\hline$C(30)$ & $10202(5)$ & $3249(4)$ & $9090(3)$ & $65(2)$ \\
\hline $\mathrm{C}(31)$ & $9454(5)$ & $3446(3)$ & $8598(3)$ & $61(2)$ \\
\hline$C(32)$ & $8373(5)$ & $3128(3)$ & $8439(2)$ & $49(1)$ \\
\hline$C(33)$ & $5392(4)$ & $593(2)$ & $6394(2)$ & $36(1)$ \\
\hline$C(34)$ & $5565(4)$ & $-141(3)$ & $6378(2)$ & $46(1)$ \\
\hline$C(35)$ & $5973(5)$ & $-455(3)$ & $5947(2)$ & $54(1)$ \\
\hline$C(36)$ & $6218(5)$ & $-41(3)$ & $5527(2)$ & $60(2)$ \\
\hline $\mathrm{C}(37)$ & $6078(5)$ & $676(3)$ & $5539(2)$ & $59(2)$ \\
\hline $\mathrm{C}(38)$ & $5655(5)$ & $1003(3)$ & $5968(2)$ & $50(1)$ \\
\hline$C(39)$ & $654(4)$ & $118(3)$ & $7609(2)$ & $42(1)$ \\
\hline $\mathrm{C}(40)$ & $395(5)$ & $-492(3)$ & $7895(2)$ & $51(1)$ \\
\hline $\mathrm{C}(41)$ & $-656(5)$ & $-834(3)$ & $7706(3)$ & $61(2)$ \\
\hline$C(42)$ & $-1455(5)$ & $-579(3)$ & $7242(3)$ & $65(2)$ \\
\hline$C(43)$ & $-1208(5)$ & $22(3)$ & $6949(3)$ & $65(2)$ \\
\hline$C(44)$ & $-165(4)$ & $373(3)$ & $7138(2)$ & $55(1)$ \\
\hline$C(45)$ & 4241(4) & $643(2)$ & $8681(2)$ & $32(1)$ \\
\hline$C(46)$ & $5237(4)$ & $1002(2)$ & $8832(2)$ & $32(1)$ \\
\hline$C(47)$ & $4126(4)$ & $-145(2)$ & $8609(2)$ & $37(1)$ \\
\hline $\mathrm{C}(48)$ & $3659(5)$ & $-529(3)$ & $8995(2)$ & $49(1)$ \\
\hline $\mathrm{C}(49)$ & $3497(6)$ & $-1254(3)$ & $8940(3)$ & $64(2)$ \\
\hline$C(50)$ & $3765(6)$ & $-1616(3)$ & $8500(3)$ & $70(2)$ \\
\hline $\mathrm{C}(51)$ & $4213(6)$ & $-1256(3)$ & $8114(3)$ & $64(2)$ \\
\hline$C(52)$ & $4405(5)$ & $-519(3)$ & $8162(2)$ & $49(1)$ \\
\hline$C(53)$ & $6397(4)$ & $651(2)$ & $8981(2)$ & $37(1)$ \\
\hline$C(54)$ & $7005(5)$ & $666(3)$ & $9543(2)$ & $52(1)$ \\
\hline$C(55)$ & $8074(5)$ & $345(4)$ & $9717(3)$ & $70(2)$ \\
\hline
\end{tabular}




$\begin{array}{lllll}\mathrm{C}(56) & 8562(5) & 21(4) & 9328(3) & 78(2) \\ \mathrm{C}(57) & 7992(6) & 7(4) & 8762(3) & 75(2) \\ \mathrm{C}(58) & 6911(5) & 322(3) & 8591(2) & 52(1) \\ \mathrm{C}(59) & 2160(4) & 2214(3) & 7588(2) & 46(1) \\ \mathrm{C}(60) & 1723(5) & 2511(3) & 8080(3) & 63(2) \\ \mathrm{C}(61) & 9466(14) & 2499(9) & 1166(8) & 301(14) \\ \mathrm{Cl}(1) & 4217(1) & 3167(1) & 7957(1) & 67(1) \\ \mathrm{Cl}(2) & 9584(3) & 1728(2) & 805(1) & 138(1) \\ \mathrm{Cl}(3) & 8412(3) & 3033(2) & 957(2) & 171(1) \\ \mathrm{N}(1) & 3134(3) & 999(2) & 8680(2) & 33(1) \\ \mathrm{N}(2) & 5263(3) & 1760(2) & 8997(2) & 34(1) \\ \mathrm{N}(3) & 5620(3) & 1663(2) & 7749(2) & 35(1) \\ \mathrm{N}(4) & 3447(3) & 928(2) & 7421(2) & 35(1) \\ \mathrm{O}(1) & 1515(4) & 2118(3) & 7128(2) & 77(1) \\ \mathrm{O}(2) & 9082(12) & 100(9) & 4999(9) & 349(11) \\ \mathrm{Pd}(1) & 3802(1) & 1975(1) & 7712(1) & 35(1)\end{array}$

Table 3. Bond lengths $[\AA ̊]$ and angles $\left[{ }^{\circ}\right]$ for (4).

$\begin{array}{ll}\mathrm{C}(1)-\mathrm{N}(1) & 1.393(5) \\ \mathrm{C}(1)-\mathrm{C}(20) & 1.400(6) \\ \mathrm{C}(1)-\mathrm{C}(2) & 1.420(6) \\ \mathrm{C}(2)-\mathrm{C}(3) & 1.353(7) \\ \mathrm{C}(3)-\mathrm{C}(4) & 1.413(6) \\ \mathrm{C}(4)-\mathrm{C}(5) & 1.392(6) \\ \mathrm{C}(4)-\mathrm{N}(1) & 1.402(5) \\ \mathrm{C}(5)-\mathrm{C}(6) & 1.401(6) \\ \mathrm{C}(5)-\mathrm{C}(21) & 1.510(6)\end{array}$




\begin{tabular}{|c|c|}
\hline $\mathrm{C}(6)-\mathrm{N}(2)$ & $1.391(5)$ \\
\hline $\mathrm{C}(6)-\mathrm{C}(7)$ & $1.412(6)$ \\
\hline $\mathrm{C}(7)-\mathrm{C}(8)$ & $1.357(7)$ \\
\hline $\mathrm{C}(8)-\mathrm{C}(9)$ & $1.400(7)$ \\
\hline $\mathrm{C}(9)-\mathrm{N}(2)$ & $1.397(6)$ \\
\hline$C(9)-C(10)$ & $1.406(6)$ \\
\hline$C(10)-C(11)$ & $1.392(6)$ \\
\hline $\mathrm{C}(10)-\mathrm{C}(27)$ & $1.483(6)$ \\
\hline $\mathrm{C}(11)-\mathrm{N}(3)$ & $1.360(6)$ \\
\hline $\mathrm{C}(11)-\mathrm{C}(12)$ & $1.451(6)$ \\
\hline$C(12)-C(13)$ & $1.339(7)$ \\
\hline $\mathrm{C}(13)-\mathrm{C}(14)$ & $1.436(7)$ \\
\hline $\mathrm{C}(14)-\mathrm{N}(3)$ & $1.364(5)$ \\
\hline$C(14)-C(15)$ & $1.394(6)$ \\
\hline$C(15)-C(16)$ & $1.417(7)$ \\
\hline$C(15)-C(33)$ & $1.501(6)$ \\
\hline $\mathrm{C}(16)-\mathrm{N}(4)$ & $1.381(6)$ \\
\hline$C(16)-C(17)$ & $1.440(7)$ \\
\hline $\mathrm{C}(17)-\mathrm{C}(18)$ & $1.337(7)$ \\
\hline$C(18)-C(19)$ & $1.443(7)$ \\
\hline $\mathrm{C}(19)-\mathrm{N}(4)$ & $1.358(6)$ \\
\hline$C(19)-C(20)$ & $1.413(6)$ \\
\hline$C(20)-C(39)$ & $1.496(6)$ \\
\hline$C(21)-C(22)$ & $1.371(8)$ \\
\hline$C(21)-C(26)$ & $1.374(8)$ \\
\hline$C(22)-C(23)$ & $1.397(9)$ \\
\hline$C(23)-C(24)$ & $1.386(11)$ \\
\hline$C(24)-C(25)$ & $1.340(10)$ \\
\hline$C(25)-C(26)$ & $1.392(8)$ \\
\hline $\mathrm{C}(27)-\mathrm{C}(32)$ & $1.380(7)$ \\
\hline
\end{tabular}




\begin{tabular}{|c|c|}
\hline$C(27)-C(28)$ & $1.405(7)$ \\
\hline$C(28)-C(29)$ & $1.381(8)$ \\
\hline$C(29)-C(30)$ & $1.373(9)$ \\
\hline $\mathrm{C}(30)-\mathrm{C}(31)$ & $1.355(9)$ \\
\hline $\mathrm{C}(31)-\mathrm{C}(32)$ & $1.381(7)$ \\
\hline $\mathrm{C}(33)-\mathrm{C}(38)$ & $1.371(7)$ \\
\hline $\mathrm{C}(33)-\mathrm{C}(34)$ & $1.386(7)$ \\
\hline $\mathrm{C}(34)-\mathrm{C}(35)$ & $1.372(7)$ \\
\hline$C(35)-C(36)$ & $1.355(8)$ \\
\hline$C(36)-C(37)$ & $1.348(8)$ \\
\hline $\mathrm{C}(37)-\mathrm{C}(38)$ & $1.389(7)$ \\
\hline $\mathrm{C}(39)-\mathrm{C}(44)$ & $1.394(7)$ \\
\hline $\mathrm{C}(39)-\mathrm{C}(40)$ & $1.399(7)$ \\
\hline $\mathrm{C}(40)-\mathrm{C}(41)$ & $1.379(7)$ \\
\hline $\mathrm{C}(41)-\mathrm{C}(42)$ & $1.370(9)$ \\
\hline$C(42)-C(43)$ & $1.392(9)$ \\
\hline $\mathrm{C}(43)-\mathrm{C}(44)$ & $1.379(7)$ \\
\hline$C(45)-C(46)$ & $1.332(6)$ \\
\hline $\mathrm{C}(45)-\mathrm{N}(1)$ & $1.469(6)$ \\
\hline $\mathrm{C}(45)-\mathrm{C}(47)$ & $1.483(6)$ \\
\hline $\mathrm{C}(46)-\mathrm{N}(2)$ & $1.466(6)$ \\
\hline$C(46)-C(53)$ & $1.487(6)$ \\
\hline $\mathrm{C}(47)-\mathrm{C}(52)$ & $1.385(7)$ \\
\hline $\mathrm{C}(47)-\mathrm{C}(48)$ & $1.388(7)$ \\
\hline $\mathrm{C}(48)-\mathrm{C}(49)$ & $1.368(8)$ \\
\hline$C(49)-C(50)$ & 1.354(9) \\
\hline$C(50)-C(51)$ & $1.352(9)$ \\
\hline $\mathrm{C}(51)-\mathrm{C}(52)$ & $1.394(8)$ \\
\hline $\mathrm{C}(53)-\mathrm{C}(54)$ & $1.377(7)$ \\
\hline $\mathrm{C}(53)-\mathrm{C}(58)$ & $1.378(7)$ \\
\hline
\end{tabular}




\begin{tabular}{|c|c|}
\hline$C(54)-C(55)$ & $1.375(8)$ \\
\hline$C(55)-C(56)$ & $1.352(9)$ \\
\hline$C(56)-C(57)$ & $1.373(9)$ \\
\hline$C(57)-C(58)$ & $1.381(8)$ \\
\hline $\mathrm{C}(59)-\mathrm{O}(1)$ & $1.203(6)$ \\
\hline$C(59)-C(60)$ & $1.504(7)$ \\
\hline $\mathrm{C}(59)-\mathrm{Pd}(1)$ & $1.949(5)$ \\
\hline $\mathrm{C}(61)-\mathrm{Cl}(3)$ & $1.585(13)$ \\
\hline $\mathrm{C}(61)-\mathrm{Cl}(2)$ & $1.703(13)$ \\
\hline $\mathrm{Cl}(1)-\mathrm{Pd}(1)$ & $2.3219(14)$ \\
\hline $\mathrm{N}(3)-\mathrm{Pd}(1)$ & $2.213(4)$ \\
\hline $\mathrm{N}(4)-\mathrm{Pd}(1)$ & $2.084(4)$ \\
\hline $\mathrm{N}(1)-\mathrm{C}(1)-\mathrm{C}(20)$ & $128.3(4)$ \\
\hline $\mathrm{N}(1)-\mathrm{C}(1)-\mathrm{C}(2)$ & $107.0(4)$ \\
\hline$C(20)-C(1)-C(2)$ & $124.7(4)$ \\
\hline $\mathrm{C}(3)-\mathrm{C}(2)-\mathrm{C}(1)$ & $108.9(4)$ \\
\hline$C(2)-C(3)-C(4)$ & $108.7(4)$ \\
\hline $\mathrm{C}(5)-\mathrm{C}(4)-\mathrm{N}(1)$ & $125.0(4)$ \\
\hline$C(5)-C(4)-C(3)$ & $126.9(4)$ \\
\hline $\mathrm{N}(1)-\mathrm{C}(4)-\mathrm{C}(3)$ & 107.1(4) \\
\hline $\mathrm{C}(4)-\mathrm{C}(5)-\mathrm{C}(6)$ & $123.7(4)$ \\
\hline $\mathrm{C}(4)-\mathrm{C}(5)-\mathrm{C}(21)$ & $117.8(4)$ \\
\hline$C(6)-C(5)-C(21)$ & $117.7(4)$ \\
\hline $\mathrm{N}(2)-\mathrm{C}(6)-\mathrm{C}(5)$ & 126.1(4) \\
\hline $\mathrm{N}(2)-\mathrm{C}(6)-\mathrm{C}(7)$ & $107.5(4)$ \\
\hline$C(5)-C(6)-C(7)$ & $126.1(4)$ \\
\hline$C(8)-C(7)-C(6)$ & $107.7(4)$ \\
\hline$C(7)-C(8)-C(9)$ & $109.8(4)$ \\
\hline $\mathrm{N}(2)-\mathrm{C}(9)-\mathrm{C}(8)$ & $106.7(4)$ \\
\hline
\end{tabular}




\begin{tabular}{|c|c|}
\hline$N(2)-C(9)-C(10)$ & $129.3(4)$ \\
\hline$C(8)-C(9)-C(10)$ & $124.0(4)$ \\
\hline$C(11)-C(10)-C(9)$ & $126.5(4)$ \\
\hline $\mathrm{C}(11)-\mathrm{C}(10)-\mathrm{C}(27)$ & $116.6(4)$ \\
\hline$C(9)-C(10)-C(27)$ & $116.9(4)$ \\
\hline $\mathrm{N}(3)-\mathrm{C}(11)-\mathrm{C}(10)$ & $127.2(4)$ \\
\hline $\mathrm{N}(3)-\mathrm{C}(11)-\mathrm{C}(12)$ & $109.0(4)$ \\
\hline $\mathrm{C}(10)-\mathrm{C}(11)-\mathrm{C}(12)$ & $123.7(4)$ \\
\hline $\mathrm{C}(13)-\mathrm{C}(12)-\mathrm{C}(11)$ & $107.0(4)$ \\
\hline $\mathrm{C}(12)-\mathrm{C}(13)-\mathrm{C}(14)$ & $107.4(4)$ \\
\hline $\mathrm{N}(3)-\mathrm{C}(14)-\mathrm{C}(15)$ & $125.8(4)$ \\
\hline $\mathrm{N}(3)-\mathrm{C}(14)-\mathrm{C}(13)$ & $109.4(4)$ \\
\hline $\mathrm{C}(15)-\mathrm{C}(14)-\mathrm{C}(13)$ & $124.4(4)$ \\
\hline$C(14)-C(15)-C(16)$ & $124.2(4)$ \\
\hline $\mathrm{C}(14)-\mathrm{C}(15)-\mathrm{C}(33)$ & $118.0(4)$ \\
\hline$C(16)-C(15)-C(33)$ & $117.2(4)$ \\
\hline $\mathrm{N}(4)-\mathrm{C}(16)-\mathrm{C}(15)$ & $125.6(4)$ \\
\hline $\mathrm{N}(4)-\mathrm{C}(16)-\mathrm{C}(17)$ & $109.0(4)$ \\
\hline$C(15)-C(16)-C(17)$ & $125.1(4)$ \\
\hline$C(18)-C(17)-C(16)$ & $107.2(4)$ \\
\hline $\mathrm{C}(17)-\mathrm{C}(18)-\mathrm{C}(19)$ & $107.6(4)$ \\
\hline $\mathrm{N}(4)-\mathrm{C}(19)-\mathrm{C}(20)$ & $127.3(4)$ \\
\hline $\mathrm{N}(4)-\mathrm{C}(19)-\mathrm{C}(18)$ & $109.3(4)$ \\
\hline$C(20)-C(19)-C(18)$ & $123.3(4)$ \\
\hline$C(1)-C(20)-C(19)$ & $127.7(4)$ \\
\hline$C(1)-C(20)-C(39)$ & $116.6(4)$ \\
\hline$C(19)-C(20)-C(39)$ & $115.7(4)$ \\
\hline $\mathrm{C}(22)-\mathrm{C}(21)-\mathrm{C}(26)$ & $119.3(5)$ \\
\hline$C(22)-C(21)-C(5)$ & $121.4(5)$ \\
\hline$C(26)-C(21)-C(5)$ & $119.3(5)$ \\
\hline
\end{tabular}




\begin{tabular}{|c|c|}
\hline$C(21)-C(22)-C(23)$ & $119.6(6)$ \\
\hline$C(24)-C(23)-C(22)$ & $120.4(7)$ \\
\hline$C(25)-C(24)-C(23)$ & $119.4(6)$ \\
\hline$C(24)-C(25)-C(26)$ & $120.8(7)$ \\
\hline$C(21)-C(26)-C(25)$ & $120.5(6)$ \\
\hline$C(32)-C(27)-C(28)$ & $118.1(5)$ \\
\hline$C(32)-C(27)-C(10)$ & $121.6(4)$ \\
\hline $\mathrm{C}(28)-\mathrm{C}(27)-\mathrm{C}(10)$ & $120.2(4)$ \\
\hline $\mathrm{C}(29)-\mathrm{C}(28)-\mathrm{C}(27)$ & $119.8(6)$ \\
\hline $\mathrm{C}(30)-\mathrm{C}(29)-\mathrm{C}(28)$ & $120.4(6)$ \\
\hline $\mathrm{C}(31)-\mathrm{C}(30)-\mathrm{C}(29)$ & $120.4(5)$ \\
\hline $\mathrm{C}(30)-\mathrm{C}(31)-\mathrm{C}(32)$ & $120.1(6)$ \\
\hline $\mathrm{C}(27)-\mathrm{C}(32)-\mathrm{C}(31)$ & $121.1(5)$ \\
\hline $\mathrm{C}(38)-\mathrm{C}(33)-\mathrm{C}(34)$ & $118.0(5)$ \\
\hline $\mathrm{C}(38)-\mathrm{C}(33)-\mathrm{C}(15)$ & $121.0(4)$ \\
\hline $\mathrm{C}(34)-\mathrm{C}(33)-\mathrm{C}(15)$ & $120.9(4)$ \\
\hline $\mathrm{C}(35)-\mathrm{C}(34)-\mathrm{C}(33)$ & $121.4(5)$ \\
\hline $\mathrm{C}(36)-\mathrm{C}(35)-\mathrm{C}(34)$ & $119.7(5)$ \\
\hline $\mathrm{C}(37)-\mathrm{C}(36)-\mathrm{C}(35)$ & $120.0(5)$ \\
\hline $\mathrm{C}(36)-\mathrm{C}(37)-\mathrm{C}(38)$ & $121.2(5)$ \\
\hline $\mathrm{C}(33)-\mathrm{C}(38)-\mathrm{C}(37)$ & $119.6(5)$ \\
\hline $\mathrm{C}(44)-\mathrm{C}(39)-\mathrm{C}(40)$ & $119.2(5)$ \\
\hline $\mathrm{C}(44)-\mathrm{C}(39)-\mathrm{C}(20)$ & $120.5(5)$ \\
\hline C(40)-C(39)-C(20) & $120.2(4)$ \\
\hline C(41)-C(40)-C(39) & $119.9(5)$ \\
\hline $\mathrm{C}(42)-\mathrm{C}(41)-\mathrm{C}(40)$ & $120.5(6)$ \\
\hline C(41)-C(42)-C(43) & $120.4(5)$ \\
\hline $\mathrm{C}(44)-\mathrm{C}(43)-\mathrm{C}(42)$ & $119.6(6)$ \\
\hline $\mathrm{C}(43)-\mathrm{C}(44)-\mathrm{C}(39)$ & $120.4(6)$ \\
\hline $\mathrm{C}(46)-\mathrm{C}(45)-\mathrm{N}(1)$ & $120.5(4$ \\
\hline
\end{tabular}




\begin{tabular}{|c|c|}
\hline $\mathrm{C}(46)-\mathrm{C}(45)-\mathrm{C}(47)$ & $125.5(4)$ \\
\hline $\mathrm{N}(1)-\mathrm{C}(45)-\mathrm{C}(47)$ & $113.0(4)$ \\
\hline $\mathrm{C}(45)-\mathrm{C}(46)-\mathrm{N}(2)$ & $121.3(4)$ \\
\hline$C(45)-C(46)-C(53)$ & $123.7(4)$ \\
\hline$N(2)-C(46)-C(53)$ & $113.5(4)$ \\
\hline $\mathrm{C}(52)-\mathrm{C}(47)-\mathrm{C}(48)$ & $117.7(5)$ \\
\hline$C(52)-C(47)-C(45)$ & $124.0(4)$ \\
\hline $\mathrm{C}(48)-\mathrm{C}(47)-\mathrm{C}(45)$ & $118.2(4)$ \\
\hline $\mathrm{C}(49)-\mathrm{C}(48)-\mathrm{C}(47)$ & $120.9(5)$ \\
\hline $\mathrm{C}(50)-\mathrm{C}(49)-\mathrm{C}(48)$ & $121.0(6)$ \\
\hline$C(51)-C(50)-C(49)$ & $119.5(6)$ \\
\hline$C(50)-C(51)-C(52)$ & $121.0(6)$ \\
\hline $\mathrm{C}(47)-\mathrm{C}(52)-\mathrm{C}(51)$ & $119.9(5)$ \\
\hline $\mathrm{C}(54)-\mathrm{C}(53)-\mathrm{C}(58)$ & $117.8(4)$ \\
\hline $\mathrm{C}(54)-\mathrm{C}(53)-\mathrm{C}(46)$ & $118.1(4)$ \\
\hline $\mathrm{C}(58)-\mathrm{C}(53)-\mathrm{C}(46)$ & $124.1(4)$ \\
\hline$C(55)-C(54)-C(53)$ & $121.7(5)$ \\
\hline$C(56)-C(55)-C(54)$ & $119.7(6)$ \\
\hline $\mathrm{C}(55)-\mathrm{C}(56)-\mathrm{C}(57)$ & $120.3(6)$ \\
\hline$C(56)-C(57)-C(58)$ & $119.7(6)$ \\
\hline $\mathrm{C}(53)-\mathrm{C}(58)-\mathrm{C}(57)$ & $120.8(5)$ \\
\hline $\mathrm{O}(1)-\mathrm{C}(59)-\mathrm{C}(60)$ & $121.2(5)$ \\
\hline $\mathrm{O}(1)-\mathrm{C}(59)-\mathrm{Pd}(1)$ & $120.3(4)$ \\
\hline $\mathrm{C}(60)-\mathrm{C}(59)-\mathrm{Pd}(1)$ & $118.6(4)$ \\
\hline $\mathrm{Cl}(3)-\mathrm{C}(61)-\mathrm{Cl}(2)$ & $121.3(7)$ \\
\hline $\mathrm{C}(1)-\mathrm{N}(1)-\mathrm{C}(4)$ & $108.3(3)$ \\
\hline $\mathrm{C}(1)-\mathrm{N}(1)-\mathrm{C}(45)$ & $123.0(3)$ \\
\hline $\mathrm{C}(4)-\mathrm{N}(1)-\mathrm{C}(45)$ & $119.0(3)$ \\
\hline $\mathrm{C}(6)-\mathrm{N}(2)-\mathrm{C}(9)$ & $108.4(4)$ \\
\hline $\mathrm{C}(6)-\mathrm{N}(2)-\mathrm{C}(46)$ & $120.0(4)$ \\
\hline
\end{tabular}




$\begin{array}{ll}\mathrm{C}(9)-\mathrm{N}(2)-\mathrm{C}(46) & 122.6(4) \\ \mathrm{C}(11)-\mathrm{N}(3)-\mathrm{C}(14) & 107.2(4) \\ \mathrm{C}(11)-\mathrm{N}(3)-\mathrm{Pd}(1) & 132.9(3) \\ \mathrm{C}(14)-\mathrm{N}(3)-\mathrm{Pd}(1) & 115.1(3) \\ \mathrm{C}(19)-\mathrm{N}(4)-\mathrm{C}(16) & 106.8(4) \\ \mathrm{C}(19)-\mathrm{N}(4)-\mathrm{Pd}(1) & 129.9(3) \\ \mathrm{C}(16)-\mathrm{N}(4)-\mathrm{Pd}(1) & 116.2(3) \\ \mathrm{C}(59)-\mathrm{Pd}(1)-\mathrm{N}(4) & 92.68(19) \\ \mathrm{C}(59)-\mathrm{Pd}(1)-\mathrm{N}(3) & 173.40(18) \\ \mathrm{N}(4)-\operatorname{Pd}(1)-\mathrm{N}(3) & 83.38(14) \\ \mathrm{C}(59)-\mathrm{Pd}(1)-\mathrm{Cl}(1) & 88.01(17) \\ \mathrm{N}(4)-\operatorname{Pd}(1)-\mathrm{Cl}(1) & 174.97(11) \\ \mathrm{N}(3)-\operatorname{Pd}(1)-\mathrm{Cl}(1) & 95.46(11)\end{array}$

Symmetry transformations used to generate equivalent atoms:

Table 4. Anisotropic displacement parameters $\left(\AA^{2} \times 10^{3}\right)$ for (4). The anisotropic displacement factor exponent takes the form: $-2 p 2\left[\mathrm{~h} 2 \mathrm{a}^{*} 2 \mathrm{U}_{11}+\ldots+2 \mathrm{~h} \mathrm{k} \mathrm{a*} \mathrm{b}^{*} \mathrm{U}_{12}\right]$

\begin{tabular}{lllllll}
\hline & $\mathrm{U}_{11}$ & $\mathrm{U}_{22}$ & $\mathrm{U}_{33}$ & $\mathrm{U}_{23}$ & $\mathrm{U}_{13}$ & $\mathrm{U}_{12}$ \\
\hline $\mathrm{C}(1)$ & $27(2)$ & $30(2)$ & $41(2)$ & $-4(2)$ & $6(2)$ & $-3(2)$ \\
$\mathrm{C}(2)$ & $27(2)$ & $51(3)$ & $51(3)$ & $-7(2)$ & $11(2)$ & $-5(2)$ \\
$\mathrm{C}(3)$ & $36(3)$ & $45(3)$ & $47(3)$ & $-9(2)$ & $18(2)$ & $-4(2)$ \\
$\mathrm{C}(4)$ & $34(2)$ & $36(3)$ & $38(2)$ & $0(2)$ & $11(2)$ & $2(2)$ \\
$\mathrm{C}(5)$ & $34(2)$ & $39(3)$ & $37(2)$ & $-7(2)$ & $9(2)$ & $-1(2)$ \\
$\mathrm{C}(6)$ & $34(2)$ & $40(3)$ & $37(2)$ & $-11(2)$ & $10(2)$ & $-5(2)$ \\
$\mathrm{C}(7)$ & $47(3)$ & $50(3)$ & $50(3)$ & $-21(2)$ & $16(2)$ & $-11(2)$ \\
$\mathrm{C}(8)$ & $43(3)$ & $50(3)$ & $57(3)$ & $-22(3)$ & $15(2)$ & $-16(2)$ \\
$\mathrm{C}(9)$ & $32(2)$ & $37(2)$ & $38(2)$ & $-7(2)$ & $5(2)$ & $-8(2)$
\end{tabular}




$\begin{array}{lllllll}\mathrm{C}(10) & 33(2) & 34(2) & 39(2) & -3(2) & 9(2) & -4(2) \\ \mathrm{C}(11) & 32(2) & 35(2) & 42(3) & 0(2) & 11(2) & -2(2) \\ \mathrm{C}(12) & 33(3) & 57(3) & 43(3) & -7(2) & 9(2) & -5(2) \\ \mathrm{C}(13) & 45(3) & 53(3) & 45(3) & -8(2) & 19(2) & -1(2) \\ \mathrm{C}(14) & 38(2) & 30(2) & 36(2) & 1(2) & 8(2) & 5(2) \\ \mathrm{C}(15) & 39(3) & 34(2) & 34(2) & -1(2) & 9(2) & 1(2) \\ \mathrm{C}(16) & 41(3) & 40(3) & 33(2) & -3(2) & 4(2) & 0(2) \\ \mathrm{C}(17) & 45(3) & 61(3) & 39(3) & -19(2) & 6(2) & -8(3) \\ \mathrm{C}(18) & 38(3) & 56(3) & 50(3) & -15(3) & 4(2) & -10(2) \\ \mathrm{C}(19) & 31(2) & 40(3) & 42(3) & -4(2) & 7(2) & -1(2) \\ \mathrm{C}(20) & 31(2) & 35(3) & 44(3) & -5(2) & 6(2) & -4(2) \\ \mathrm{C}(21) & 29(2) & 52(3) & 38(2) & -13(2) & 7(2) & -6(2) \\ \mathrm{C}(22) & 60(4) & 72(4) & 53(3) & -2(3) & 19(3) & 7(3) \\ \mathrm{C}(23) & 78(5) & 131(7) & 46(4) & 1(4) & 27(3) & 0(5) \\ \mathrm{C}(24) & 70(4) & 125(7) & 55(4) & -41(4) & 32(3) & -14(4) \\ \mathrm{C}(25) & 56(4) & 76(5) & 103(6) & -49(4) & 28(4) & -6(3) \\ \mathrm{C}(26) & 48(3) & 62(4) & 60(3) & -11(3) & 16(3) & -1(3) \\ \mathrm{C}(27) & 33(2) & 42(3) & 43(3) & -7(2) & 11(2) & -4(2) \\ \mathrm{C}(28) & 43(3) & 59(4) & 59(3) & -4(3) & 10(3) & -10(3) \\ \mathrm{C}(29) & 47(3) & 83(4) & 62(4) & -13(3) & -2(3) & -5(3) \\ \mathrm{C}(30) & 36(3) & 69(4) & 87(5) & -14(3) & 11(3) & -13(3) \\ \mathrm{C}(31) & 49(3) & 46(3) & 93(5) & 2(3) & 29(3) & -11(3) \\ \mathrm{C}(32) & 43(3) & 48(3) & 58(3) & 0(2) & 14(2) & -2(2) \\ \mathrm{C}(33) & 39(3) & 38(3) & 32(2) & -3(2) & 8(2) & 1(2) \\ \mathrm{C}(34) & 48(3) & 41(3) & 49(3) & -2(2) & 8(2) & 0(2) \\ \mathrm{C}(35) & 63(4) & 41(3) & 56(3) & -13(3) & 9(3) & 8(3) \\ \mathrm{C}(36) & 66(4) & 70(4) & 47(3) & -15(3) & 19(3) & 7(3) \\ \mathrm{C}(3) & 77(4) & 69(4) & 38(3) & 4(3) & 25(3) & 4(3) \\ \mathrm{C}(3) & 44(3) & 41(3) & 3(2) & 14(2) & 4(3) \\ \mathrm{C}(3) & 43(3) & 47(3) & -11(2) & 9(2) & -5(2)\end{array}$




$\begin{array}{lllllll}\mathrm{C}(40) & 48(3) & 57(3) & 49(3) & -6(3) & 14(2) & -13(3) \\ \mathrm{C}(41) & 54(3) & 56(4) & 73(4) & -15(3) & 18(3) & -20(3) \\ \mathrm{C}(42) & 38(3) & 62(4) & 95(5) & -36(4) & 15(3) & -13(3) \\ \mathrm{C}(43) & 35(3) & 62(4) & 87(4) & -25(3) & -9(3) & 7(3) \\ \mathrm{C}(44) & 42(3) & 48(3) & 69(4) & -9(3) & -1(3) & -1(2) \\ \mathrm{C}(45) & 34(2) & 31(2) & 31(2) & -1(2) & 8(2) & 0(2) \\ \mathrm{C}(46) & 35(2) & 33(2) & 30(2) & -1(2) & 9(2) & 4(2) \\ \mathrm{C}(47) & 32(2) & 32(2) & 43(3) & -4(2) & 3(2) & 2(2) \\ \mathrm{C}(48) & 58(3) & 41(3) & 48(3) & 1(2) & 16(2) & -3(2) \\ \mathrm{C}(49) & 80(4) & 42(3) & 70(4) & 11(3) & 19(3) & -10(3) \\ \mathrm{C}(50) & 85(5) & 36(3) & 85(5) & -3(3) & 12(4) & -3(3) \\ \mathrm{C}(51) & 76(4) & 55(4) & 59(4) & -16(3) & 7(3) & 14(3) \\ \mathrm{C}(52) & 55(3) & 43(3) & 46(3) & -2(2) & 10(2) & 8(2) \\ \mathrm{C}(53) & 31(2) & 35(2) & 44(3) & 2(2) & 8(2) & 2(2) \\ \mathrm{C}(54) & 50(3) & 56(3) & 47(3) & 0(2) & 5(2) & 6(3) \\ \mathrm{C}(55) & 55(4) & 75(4) & 65(4) & -3(3) & -14(3) & 14(3) \\ \mathrm{C}(56) & 41(3) & 84(5) & 103(6) & 13(4) & 6(3) & 25(3) \\ \mathrm{C}(57) & 62(4) & 85(5) & 83(5) & -7(4) & 30(4) & 27(4) \\ \mathrm{C}(58) & 51(3) & 57(3) & 48(3) & -4(3) & 13(2) & 7(3) \\ \mathrm{C}(59) & 44(3) & 42(3) & 53(3) & 12(2) & 13(2) & 14(2) \\ \mathrm{C}(60) & 70(4) & 52(4) & 75(4) & 10(3) & 33(3) & 24(3) \\ \mathrm{C}(61) & 210(16) & 280(20) & 300(20) & -198(18) & -173(15) & 120(15) \\ \mathrm{Cl}(1) & 69(1) & 35(1) & 101(1) & -12(1) & 27(1) & -3(1) \\ \mathrm{Cl}(2) & 161(3) & 128(2) & 126(2) & 7(2) & 34(2) & 12(2) \\ \mathrm{N}(3) & 146(3) & 171(3) & 180(3) & -27(2) & 4(2) & 43(2) \\ \mathrm{N}(2) & 31(2) & 34(2) & 35(2) & -7(2) & 9(2) & -2(2) \\ \mathrm{N}(3) & 33(2) & 37(2) & 37(2) & -5(2) & 9(2) & -2(2) \\ \mathrm{N}(2) & 37(2) & 33(2) & -6(2) & 6(2) & -1(2) \\ \mathrm{N}(2) & 110(4) & 66(3) & 14(3) & 3(2) & 18(2)\end{array}$




$\begin{array}{lllllll}\mathrm{O}(2) & 225(15) & 300(18) & 540(30) & 232(19) & 136(18) & 50(13) \\ \mathrm{Pd}(1) & 37(1) & 32(1) & 37(1) & -1(1) & 8(1) & 2(1)\end{array}$

Table 5. Hydrogen coordinates ( $\left.\times 10^{4}\right)$ and isotropic displacement parameters $\left(\AA^{2} \times 10^{3}\right)$ for (4).

\begin{tabular}{|c|c|c|c|c|}
\hline & $\mathrm{x}$ & $\mathrm{y}$ & $\mathrm{z}$ & $\mathrm{U}(\mathrm{eq})$ \\
\hline $\mathrm{H}(2)$ & 467 & 734 & 8596 & 51 \\
\hline $\mathrm{H}(3)$ & 1433 & 1314 & 9487 & 50 \\
\hline $\mathrm{H}(7)$ & 5361 & 2788 & 10049 & 58 \\
\hline $\mathrm{H}(8)$ & 6868 & 3041 & 9537 & 59 \\
\hline $\mathrm{H}(12)$ & 8308 & 1917 & 7825 & 53 \\
\hline $\mathrm{H}(13)$ & 7341 & 1276 & 6960 & 56 \\
\hline $\mathrm{H}(17)$ & 3252 & -19 & 6299 & 58 \\
\hline $\mathrm{H}(18)$ & 1654 & -224 & 6760 & 59 \\
\hline $\mathrm{H}(22)$ & 3609 & 1110 & 10554 & 72 \\
\hline $\mathrm{H}(23)$ & 3096 & 1611 & 11346 & 99 \\
\hline $\mathrm{H}(24)$ & 2610 & 2815 & 11349 & 96 \\
\hline $\mathrm{H}(25)$ & 2596 & 3496 & 10567 & 92 \\
\hline $\mathrm{H}(26)$ & 3066 & 3003 & 9769 & 68 \\
\hline $\mathrm{H}(28)$ & 8636 & 2036 & 9504 & 65 \\
\hline $\mathrm{H}(29)$ & 10425 & 2591 & 9765 & 80 \\
\hline $\mathrm{H}(30)$ & 10923 & 3472 & 9199 & 77 \\
\hline $\mathrm{H}(31)$ & 9668 & 3795 & 8366 & 73 \\
\hline $\mathrm{H}(32)$ & 7861 & 3271 & 8103 & 59 \\
\hline $\mathrm{H}(34)$ & 5400 & -428 & 6665 & 56 \\
\hline $\mathrm{H}(35)$ & 6080 & -948 & 5943 & 65 \\
\hline $\mathrm{H}(36)$ & 6484 & -252 & 5232 & 72 \\
\hline
\end{tabular}




\begin{tabular}{|c|c|c|c|c|}
\hline $\mathrm{H}(37)$ & 6268 & 956 & 5255 & 71 \\
\hline $\mathrm{H}(38)$ & 5550 & 1497 & 5966 & 59 \\
\hline $\mathrm{H}(40)$ & 931 & -665 & 8213 & 61 \\
\hline $\mathrm{H}(41)$ & -824 & -1241 & 7894 & 73 \\
\hline $\mathrm{H}(42)$ & -2167 & -809 & 7121 & 78 \\
\hline $\mathrm{H}(43)$ & -1744 & 187 & 6628 & 78 \\
\hline $\mathrm{H}(44)$ & -6 & 783 & 6949 & 66 \\
\hline $\mathrm{H}(48)$ & 3453 & -290 & 9296 & 59 \\
\hline $\mathrm{H}(49)$ & 3197 & -1502 & 9209 & 77 \\
\hline $\mathrm{H}(50)$ & 3642 & -2108 & 8465 & 84 \\
\hline $\mathrm{H}(51)$ & 4397 & -1505 & 7811 & 77 \\
\hline $\mathrm{H}(52)$ & 4720 & -279 & 7895 & 58 \\
\hline $\mathrm{H}(54)$ & 6684 & 899 & 9812 & 62 \\
\hline $\mathrm{H}(55)$ & 8461 & 351 & 10101 & 83 \\
\hline $\mathrm{H}(56)$ & 9287 & -195 & 9445 & 93 \\
\hline $\mathrm{H}(57)$ & 8332 & -213 & 8495 & 90 \\
\hline $\mathrm{H}(58)$ & 6525 & 311 & 8207 & 62 \\
\hline $\mathrm{H}(60 \mathrm{~A})$ & 906 & 2606 & 7958 & 94 \\
\hline $\mathrm{H}(60 \mathrm{~B})$ & 2127 & 2948 & 8210 & 94 \\
\hline $\mathrm{H}(60 \mathrm{C})$ & 1858 & 2169 & 8387 & 94 \\
\hline $\mathrm{H}(61 \mathrm{~A})$ & 9464 & 2363 & 1555 & 362 \\
\hline $\mathrm{H}(61 \mathrm{~B})$ & 10172 & 2771 & 1185 & 362 \\
\hline $\mathrm{H}(62 \mathrm{~A})$ & $8600(30)$ & $366(18)$ & $4795(13)$ & $0(8)$ \\
\hline $\mathrm{H}(62 \mathrm{~B})$ & $10260(40)$ & $390(20)$ & $5469(15)$ & $16(9$ \\
\hline
\end{tabular}

Table 6. Least-squares planes ( $\mathrm{x}, \mathrm{y}, \mathrm{z}$ in crystal coordinates) and deviations from them (* indicates atom used to define plane) for (4). 
PLANE 1

$5.2644(0.0137) \mathrm{x}-16.2279(0.0122) \mathrm{y}+2.4899(0.0290) \mathrm{z}=2.1728(0.0238)$

* $0.0171(0.0019) \mathrm{N} 1$

* $-0.0172(0.0019) \mathrm{N} 2$

* $\quad 0.0170(0.0018) \mathrm{N} 3$

* $-0.0170(0.0018) \mathrm{N} 4$

$-1.4567(0.0022) \operatorname{Pd} 1$

Rms deviation of fitted atoms $=0.0171$

\section{PLANE 2}

$-1.4634(0.0201) x-5.8301(0.0303) y+22.7399(0.0131) z=15.8293(0.0166)$

Angle to previous plane (with approximate esd) $=65.32(0.13)$

* $0.0000(0.0000) \mathrm{N} 3$

* $\quad 0.0000(0.0000) \mathrm{N} 4$

* $0.0000(0.0000) \mathrm{Pd} 1$

Rms deviation of fitted atoms $=0.0000$

PLANE 3

$4.4043(0.0106) x-15.8557(0.0097) y+6.6388(0.0214) z=5.2518(0.0176)$

Angle to previous plane (with approximate esd) $=55.19(0.12)$

* $0.0418(0.0023)$ C5

* $-0.0423(0.0023) \mathrm{C} 10$

* $0.0428(0.0023) \mathrm{C} 15$

* $-0.0422(0.0023)$ C20

$-1.5895(0.0024) \operatorname{Pd} 1$

Rms deviation of fitted atoms $=0.0423$

\section{PLANE 4}

$-1.8564(0.0182) \mathrm{x}-4.9119(0.0165) \mathrm{y}+23.1617(0.0070) \mathrm{z}=16.0899(0.0095)$

Angle to previous plane (with approximate esd) $=58.64(0.09)$ 
* $-0.0019(0.0018) \mathrm{N} 3$

* $\quad 0.0022(0.0020) \mathrm{N} 4$

* $-0.0021(0.0020)$ C59

* $0.0019(0.0017) \mathrm{Cl} 1$

$0.0967(0.0019) \operatorname{Pd} 1$

Rms deviation of fitted atoms $=0.0020$

\section{PLANE 5}

$1.3600(0.0281) x-16.7310(0.0205) y+9.3430(0.0538) z=6.8474(0.0480)$

Angle to the plane 3 (with approximate esd) $=15.33(0.20)$

* $\quad 0.0172(0.0027) \mathrm{N} 1$

* $-0.0146(0.0028) \mathrm{C} 1$

* $0.0065(0.0031) \mathrm{C} 2$

* $0.0040(0.0031) \mathrm{C} 3$

* $-0.0131(0.0028) \mathrm{C} 4$

Rms deviation of fitted atoms $=0.0121$

PLANE 6

$5.7378(0.0278) x-11.9593(0.0380) y+11.2113(0.0554) z=10.9919(0.0497)$

Angle to the plane 3 (with approximate esd) $=18.72(0.26)$

* $\quad 0.0107(0.0028) \mathrm{N} 2$

* $-0.0098(0.0030)$ C6

* $0.0053(0.0033) \mathrm{C} 7$

* $0.0012(0.0034) \mathrm{C} 8$

* $-0.0073(0.0030)$ C9

Rms deviation of fitted atoms $=0.0077$

PLANE 7

$1.2311(0.0289) \mathrm{x}-16.2638(0.0235) \mathrm{y}+10.6121(0.0547) \mathrm{z}=6.2219(0.0445)$

Angle to the plane 3 (with approximate esd) $=16.60(0.21)$ 
* $-0.0109(0.0027) \mathrm{N} 3$

* $0.0092(0.0029) \mathrm{C} 11$

* $-0.0041(0.0032) \mathrm{C} 12$

* $-0.0022(0.0032) \mathrm{C} 13$

* $0.0080(0.0029) \mathrm{C} 14$

$-0.7822(0.0078) \operatorname{Pd} 1$

Rms deviation of fitted atoms $=0.0076$

PLANE 8

$4.9714(0.0286) \mathrm{x}-13.5480(0.0319) \mathrm{y}+10.3829(0.0552) \mathrm{z}=8.1852(0.0365)$

Angle to the plane 3 (with approximate esd $)=12.44(0.26)$

* $-0.0243(0.0028) \mathrm{N} 4$

* 0.0155 (0.0029) $\mathrm{C} 16$

* $-0.0010(0.0033) \mathrm{C} 17$

* $-0.0134(0.0033) \mathrm{C} 18$

* $0.0232(0.0030) \mathrm{C} 19$

$-0.9639(0.0073)$ Pd1

Rms deviation of fitted atoms $=0.0176$

Table 7. Crystal data and structure refinement for (5).

Identification code

Empirical formula

Formula weight

Temperature

Wavelength

Crystal system

Space group (por) $\mathrm{Pd}(\mathrm{Me})(\mathrm{CO}) \mathrm{ClO}_{4} \cdot \mathrm{C}_{4} \mathrm{H}_{10} \mathrm{O}$

C64 H51 Cl N4 O6 Pd

1113.94

135(2) K

$0.71073 \AA$

triclinic

$\mathrm{P}-1$ 


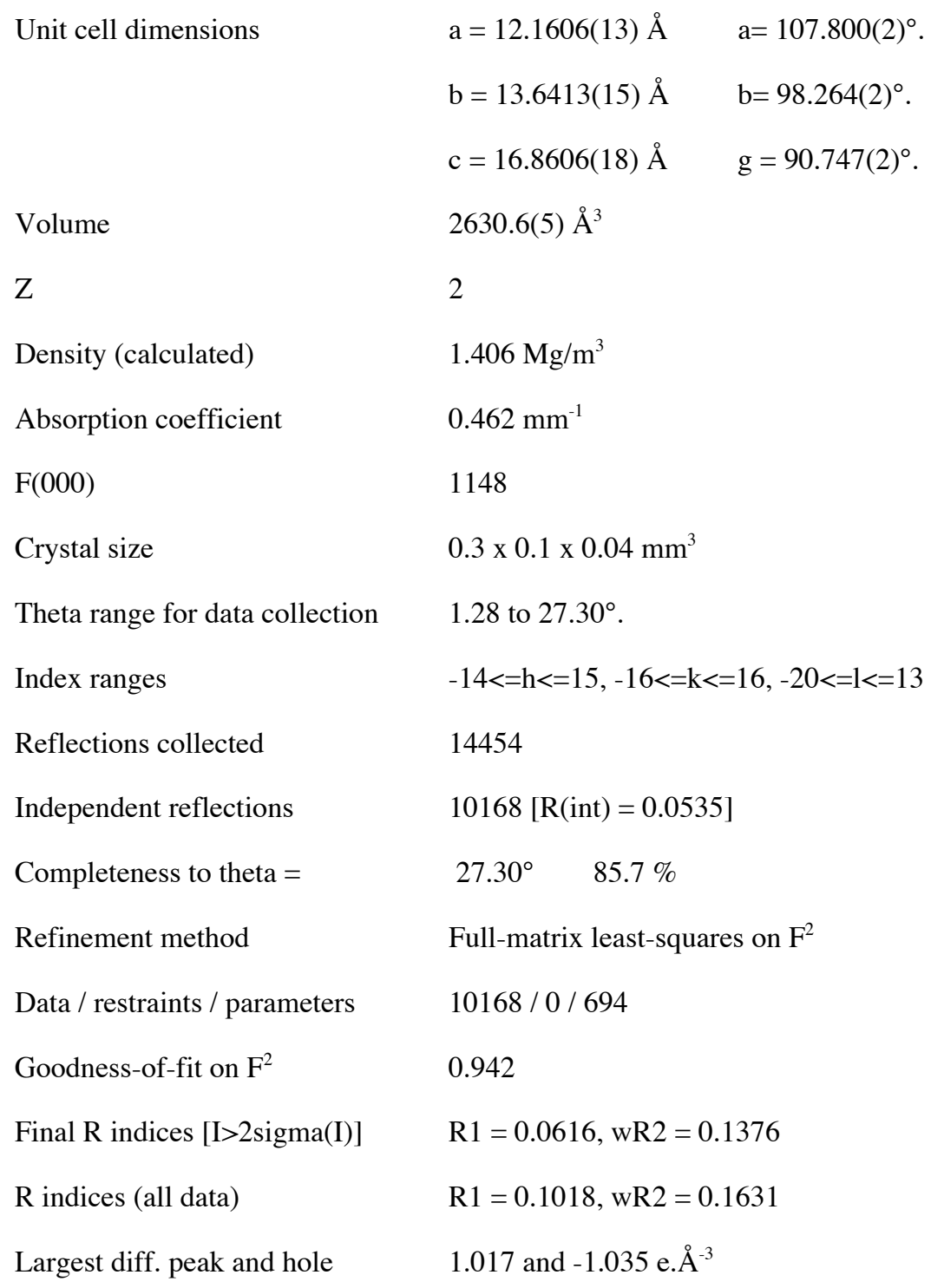

Table 8. Atomic coordinates $\left(\times 10^{4}\right)$ and equivalent isotropic displacement parameters $\left(\AA^{2} \times 10^{3}\right)$ for $(\mathbf{5})$. U(eq) is defined as one third of the trace of the orthogonalized Uij tensor.

\begin{tabular}{lllll}
\hline & $\mathrm{x}$ & $\mathrm{y}$ & $\mathrm{z}$ & $\mathrm{U}(\mathrm{eq})$ \\
$\mathrm{C}(1)$ & $3455(4)$ & $8385(4)$ & $6888(3)$ & $23(1)$ \\
$\mathrm{C}(2)$ & $2326(4)$ & $8477(4)$ & $6992(3)$ & $32(1)$ \\
$\mathrm{C}(3)$ & $2222(4)$ & $9371(4)$ & $7602(3)$ & $31(1)$
\end{tabular}




\begin{tabular}{|c|c|c|c|c|}
\hline $\mathrm{C}(4)$ & $3283(4)$ & 9891(4) & $7886(3)$ & $25(1)$ \\
\hline$C(5)$ & $3495(4)$ & $10918(4)$ & $8395(3)$ & $24(1)$ \\
\hline$C(6)$ & $4441(4)$ & $11512(4)$ & $8407(3)$ & $23(1)$ \\
\hline $\mathrm{C}(7)$ & $4556(4)$ & $12595(4)$ & $8667(3)$ & $29(1)$ \\
\hline $\mathrm{C}(8)$ & $5535(4)$ & $12876(4)$ & $8467(3)$ & $27(1)$ \\
\hline $\mathrm{C}(9)$ & $6081(4)$ & $11973(4)$ & $8090(3)$ & $22(1)$ \\
\hline$C(10)$ & $7080(4)$ & 11983(4) & $7784(3)$ & $24(1)$ \\
\hline $\mathrm{C}(11)$ & $7521(4)$ & $11165(4)$ & $7195(3)$ & $25(1)$ \\
\hline $\mathrm{C}(12)$ & $8653(5)$ & $11135(4)$ & $7057(4)$ & $34(1)$ \\
\hline $\mathrm{C}(13)$ & $8764(4)$ & $10232(4)$ & $6472(4)$ & $33(1)$ \\
\hline$C(14)$ & $7705(4)$ & $9669(4)$ & $6264(3)$ & $26(1)$ \\
\hline$C(15)$ & $7477(4)$ & $8635(4)$ & $5754(3)$ & $23(1)$ \\
\hline$C(16)$ & $6533(4)$ & $8040(4)$ & $5752(3)$ & $23(1)$ \\
\hline$C(17)$ & $6418(4)$ & 6931(4) & $5455(3)$ & $27(1)$ \\
\hline $\mathrm{C}(18)$ & $5451(4)$ & $6640(4)$ & $5618(3)$ & $26(1)$ \\
\hline$C(19)$ & 4924(4) & $7579(4)$ & $6021(3)$ & $23(1)$ \\
\hline$C(20)$ & $3873(4)$ & $7582(4)$ & $6278(3)$ & $23(1)$ \\
\hline $\mathrm{C}(21)$ & $2575(4)$ & $11455(4)$ & $8810(3)$ & $23(1)$ \\
\hline $\mathrm{C}(22)$ & 2391(4) & 11391(4) & $9586(3)$ & $30(1)$ \\
\hline$C(23)$ & $1539(5)$ & $11908(5)$ & $9970(4)$ & $36(1)$ \\
\hline $\mathrm{C}(24)$ & $872(4)$ & $12478(4)$ & $9592(4)$ & $33(1)$ \\
\hline $\mathrm{C}(25)$ & $1033(4)$ & $12548(4)$ & $8814(4)$ & $33(1)$ \\
\hline$C(26)$ & $1878(5)$ & $12037(5)$ & $8428(4)$ & $38(1)$ \\
\hline $\mathrm{C}(27)$ & $7759(4)$ & $12978(4)$ & $8106(3)$ & $26(1)$ \\
\hline$C(28)$ & $8069(4)$ & $13458(4)$ & $8968(3)$ & $30(1)$ \\
\hline C(29) & $8663(5)$ & $14400(4)$ & $9264(4)$ & $37(1)$ \\
\hline $\mathrm{C}(30)$ & $8985(5)$ & $14877(4)$ & $8715(4)$ & $38(1)$ \\
\hline $\mathrm{C}(31)$ & $8706(5)$ & $14400(4)$ & $7863(4)$ & $36(1)$ \\
\hline $\mathrm{C}(32)$ & $8106(4)$ & $13462(4)$ & $7552(3)$ & $29(1)$ \\
\hline C(33) & $8330(4)$ & $8130(4)$ & $5235(3)$ & $28(1)$ \\
\hline
\end{tabular}




\begin{tabular}{|c|c|c|c|c|}
\hline $\mathrm{C}(34)$ & $9306(5)$ & $7811(5)$ & $5555(4)$ & $45(2)$ \\
\hline $\mathrm{C}(35)$ & $10061(5)$ & $7351(5)$ & $5049(4)$ & $46(2)$ \\
\hline$C(36)$ & $9870(6)$ & $7202(6)$ & 4221(4) & $54(2)$ \\
\hline $\mathrm{C}(37)$ & $8867(9)$ & $7433(10)$ & $3862(5)$ & $128(5)$ \\
\hline $\mathrm{C}(38)$ & $8114(7)$ & 7924(9) & $4368(5)$ & $105(4)$ \\
\hline C(39) & 3091(4) & $6671(4)$ & $5807(3)$ & $26(1)$ \\
\hline $\mathrm{C}(40)$ & 2971(4) & $6323(4)$ & 4918(3) & $28(1)$ \\
\hline $\mathrm{C}(41)$ & $2238(4)$ & $5490(4)$ & 4453(4) & $33(1)$ \\
\hline $\mathrm{C}(42)$ & $1595(5)$ & $5015(4)$ & $4865(4)$ & $38(2)$ \\
\hline$C(43)$ & 1709(4) & $5349(4)$ & $5731(4)$ & $36(1)$ \\
\hline $\mathrm{C}(44)$ & $2456(4)$ & $6174(4)$ & $6196(4)$ & $31(1)$ \\
\hline $\mathrm{C}(45)$ & $5179(4)$ & $9303(4)$ & 7883(3) & $21(1)$ \\
\hline $\mathrm{C}(46)$ & 5814(4) & $10180(4)$ & $8174(3)$ & $22(1)$ \\
\hline $\mathrm{C}(47)$ & $5487(4)$ & $8349(4)$ & $8108(3)$ & $25(1)$ \\
\hline $\mathrm{C}(48)$ & $6393(5)$ & $7807(4)$ & 7884(3) & $32(1)$ \\
\hline C(49) & $6647(6)$ & $6965(5)$ & $8145(4)$ & $50(2)$ \\
\hline $\mathrm{C}(51)$ & $5072(5)$ & $7186(4)$ & $8863(3)$ & $57(2)$ \\
\hline $\mathrm{C}(50)$ & $5990(5)$ & $6660(4)$ & $8650(3)$ & $57(2)$ \\
\hline$C(52)$ & $4807(5)$ & $8026(5)$ & $8596(4)$ & $35(1)$ \\
\hline$C(53)$ & $6863(4)$ & $10374(4)$ & $8787(3)$ & $20(1)$ \\
\hline $\mathrm{C}(54)$ & $6827(5)$ & $11034(4)$ & $9606(3)$ & $29(1)$ \\
\hline$C(55)$ & $7775(5)$ & $11297(4)$ & 10190(3) & $35(1)$ \\
\hline$C(56)$ & $8776(5)$ & $10945(4)$ & $9986(4)$ & $37(1)$ \\
\hline$C(57)$ & $8831(5)$ & $10293(5)$ & $9183(4)$ & $38(1)$ \\
\hline $\mathrm{C}(58)$ & $7878(4)$ & $10004(4)$ & $8583(3)$ & $28(1)$ \\
\hline C(59) & $3714(6)$ & $9636(5)$ & $5609(4)$ & $40(2)$ \\
\hline$C(60)$ & $5029(5)$ & $11368(6)$ & $6134(4)$ & $40(2)$ \\
\hline$C(61)$ & $2717(13)$ & $5002(14)$ & 7993(9) & $180(8)$ \\
\hline$C(62)$ & 2591(14) & $5250(11)$ & $8827(8)$ & $186(8)$ \\
\hline $\mathrm{C}(63)$ & $3996(16)$ & $4452(17)$ & $7089(8)$ & $317(19)$ \\
\hline
\end{tabular}




$\begin{array}{lllll}\mathrm{C}(64) & 5171(13) & 4380(8) & 6926(7) & 139(6) \\ \mathrm{Cl}(1) & 9873(2) & 7156(1) & 7694(1) & 60(1) \\ \mathrm{N}(1) & 4052(3) & 9272(3) & 7463(2) & 19(1) \\ \mathrm{N}(2) & 5383(3) & 11127(3) & 8062(2) & 20(1) \\ \mathrm{N}(3) & 6956(3) & 10259(3) & 6696(3) & 24(1) \\ \mathrm{N}(4) & 5609(3) & 8405(3) & 6096(2) & 21(1) \\ \mathrm{O}(2) & 9726(5) & 7028(4) & 8452(3) & 69(2) \\ \mathrm{O}(3) & 9866(5) & 8195(4) & 7710(3) & 69(2) \\ \mathrm{O}(4) & 10855(13) & 6835(8) & 7490(11) & 293(10) \\ \mathrm{O}(5) & 9106(13) & 6551(8) & 7086(4) & 262(9) \\ \mathrm{O}(6) & 3757(8) & 4757(6) & 7817(5) & 114(3) \\ \mathrm{O}(1 \mathrm{~A}) & 2866(6) & 9601(6) & 5397(5) & 51(3) \\ \mathrm{O}(1 \mathrm{~B}) & 4800(11) & 12003(10) & 6046(8) & 64(5) \\ \mathrm{Pd}(1) & 5267(1) & 9936(1) & 6164(1) & 23(1)\end{array}$

Table 9. Bond lengths $[\AA]$ and angles $\left[{ }^{\circ}\right]$ for $(\mathbf{5})$.

\begin{tabular}{ll}
\hline $\mathrm{C}(1)-\mathrm{C}(20)$ & $1.411(6)$ \\
$\mathrm{C}(1)-\mathrm{N}(1)$ & $1.411(6)$ \\
$\mathrm{C}(1)-\mathrm{C}(2)$ & $1.411(7)$ \\
$\mathrm{C}(2)-\mathrm{C}(3)$ & $1.354(7)$ \\
$\mathrm{C}(3)-\mathrm{C}(4)$ & $1.411(7)$ \\
$\mathrm{C}(4)-\mathrm{N}(1)$ & $1.393(6)$ \\
$\mathrm{C}(4)-\mathrm{C}(5)$ & $1.399(7)$ \\
$\mathrm{C}(5)-\mathrm{C}(6)$ & $1.392(7)$ \\
$\mathrm{C}(5)-\mathrm{C}(21)$ & $1.486(6)$ \\
$\mathrm{C}(6)-\mathrm{N}(2)$ & $1.395(6)$
\end{tabular}




$\begin{array}{ll}\mathrm{C}(6)-\mathrm{C}(7) & 1.406(7) \\ \mathrm{C}(7)-\mathrm{C}(8) & 1.363(7) \\ \mathrm{C}(8)-\mathrm{C}(9) & 1.423(7) \\ \mathrm{C}(9)-\mathrm{C}(10) & 1.387(7) \\ \mathrm{C}(9)-\mathrm{N}(2) & 1.409(6) \\ \mathrm{C}(10)-\mathrm{C}(11) & 1.418(7) \\ \mathrm{C}(10)-\mathrm{C}(27) & 1.486(7) \\ \mathrm{C}(11)-\mathrm{N}(3) & 1.373(6) \\ \mathrm{C}(11)-\mathrm{C}(12) & 1.428(7) \\ \mathrm{C}(12)-\mathrm{C}(13) & 1.345(7) \\ \mathrm{C}(13)-\mathrm{C}(14) & 1.432(7) \\ \mathrm{C}(14)-\mathrm{N}(3) & 1.364(6) \\ \mathrm{C}(14)-\mathrm{C}(15) & 1.408(7) \\ \mathrm{C}(15)-\mathrm{C}(16) & 1.397(7) \\ \mathrm{C}(15)-\mathrm{C}(33) & 1.493(6) \\ \mathrm{C}(16)-\mathrm{N}(4) & 1.393(7) \\ \mathrm{C}(16)-\mathrm{C}(17) & 1.369(6) \\ \mathrm{C}(17)-\mathrm{C}(18) & 1.439(7) \\ \mathrm{C}(18)-\mathrm{C}(19) & 1.327(7) \\ \mathrm{C}(19)-\mathrm{N}(4) & 1.459(7) \\ \mathrm{C}(19)-\mathrm{C}(20) & 1.356(6) \\ \mathrm{C}(20)-\mathrm{C}(39) & 1.406(7) \\ \mathrm{C}(21)-\mathrm{C}(22) & 1.493(7) \\ \mathrm{C}(21)-\mathrm{C}(26) & \\ \mathrm{C}(22)-\mathrm{C}(23) & \\ \mathrm{C}(23)-\mathrm{C}(24) & \\ \mathrm{C}(24)-\mathrm{C}(25) & \\ \mathrm{C}(25)-\mathrm{C}(26) & \\ & 1.387(7) \\ & \end{array}$




\begin{tabular}{|c|c|}
\hline$C(28)-C(29)$ & $1.379(8)$ \\
\hline$C(29)-C(30)$ & $1.380(9)$ \\
\hline$C(30)-C(31)$ & $1.372(8)$ \\
\hline $\mathrm{C}(31)-\mathrm{C}(32)$ & $1.379(8)$ \\
\hline $\mathrm{C}(33)-\mathrm{C}(34)$ & $1.364(7)$ \\
\hline $\mathrm{C}(33)-\mathrm{C}(38)$ & $1.385(9)$ \\
\hline $\mathrm{C}(34)-\mathrm{C}(35)$ & $1.370(8)$ \\
\hline$C(35)-C(36)$ & $1.333(9)$ \\
\hline$C(36)-C(37)$ & $1.367(10)$ \\
\hline $\mathrm{C}(37)-\mathrm{C}(38)$ & $1.381(10)$ \\
\hline$C(39)-C(44)$ & $1.375(8)$ \\
\hline $\mathrm{C}(39)-\mathrm{C}(40)$ & $1.411(7)$ \\
\hline $\mathrm{C}(40)-\mathrm{C}(41)$ & $1.389(8)$ \\
\hline$C(41)-C(42)$ & $1.389(9)$ \\
\hline$C(42)-C(43)$ & $1.376(8)$ \\
\hline $\mathrm{C}(43)-\mathrm{C}(44)$ & $1.391(8)$ \\
\hline$C(45)-C(46)$ & $1.334(7)$ \\
\hline $\mathrm{C}(45)-\mathrm{N}(1)$ & $1.443(6)$ \\
\hline$C(45)-C(47)$ & $1.500(7)$ \\
\hline $\mathrm{C}(46)-\mathrm{N}(2)$ & $1.455(6)$ \\
\hline$C(46)-C(53)$ & $1.487(6)$ \\
\hline $\mathrm{C}(47)-\mathrm{C}(48)$ & $1.369(7)$ \\
\hline $\mathrm{C}(47)-\mathrm{C}(52)$ & $1.400(8)$ \\
\hline $\mathrm{C}(48)-\mathrm{C}(49)$ & $1.375(8)$ \\
\hline $\mathrm{C}(49)-\mathrm{C}(50)$ & $1.392(9)$ \\
\hline $\mathrm{C}(51)-\mathrm{C}(50)$ & 1.3672 \\
\hline $\mathrm{C}(51)-\mathrm{C}(52)$ & $1.380(7)$ \\
\hline $\mathrm{C}(53)-\mathrm{C}(58)$ & $1.388(7)$ \\
\hline $\mathrm{C}(53)-\mathrm{C}(54)$ & $1.405(7)$ \\
\hline $\mathrm{C}(54)-\mathrm{C}(55)$ & $1.370(7)$ \\
\hline
\end{tabular}




\begin{tabular}{|c|c|}
\hline$C(55)-C(56)$ & $1.366(8)$ \\
\hline $\mathrm{C}(56)-\mathrm{C}(57)$ & $1.386(8)$ \\
\hline $\mathrm{C}(57)-\mathrm{C}(58)$ & $1.389(7)$ \\
\hline $\mathrm{C}(59)-\mathrm{O}(1 \mathrm{~A})$ & $1.037(8)$ \\
\hline $\mathrm{C}(59)-\operatorname{Pd}(1)$ & $1.961(7)$ \\
\hline $\mathrm{C}(60)-\mathrm{O}(1 \mathrm{~B})$ & $0.961(11)$ \\
\hline $\mathrm{C}(60)-\operatorname{Pd}(1)$ & $1.994(8)$ \\
\hline $\mathrm{C}(61)-\mathrm{O}(6)$ & $1.363(14)$ \\
\hline$C(61)-C(62)$ & $1.374(16)$ \\
\hline $\mathrm{C}(63)-\mathrm{O}(6)$ & $1.248(14)$ \\
\hline$C(63)-C(64)$ & $1.49(2)$ \\
\hline $\mathrm{Cl}(1)-\mathrm{O}(4)$ & $1.333(9)$ \\
\hline $\mathrm{Cl}(1)-\mathrm{O}(5)$ & $1.338(8)$ \\
\hline $\mathrm{Cl}(1)-\mathrm{O}(2)$ & $1.377(5)$ \\
\hline $\mathrm{Cl}(1)-\mathrm{O}(3)$ & $1.410(5)$ \\
\hline $\mathrm{N}(3)-\mathrm{Pd}(1)$ & $2.103(4)$ \\
\hline $\mathrm{N}(4)-\operatorname{Pd}(1)$ & $2.106(4)$ \\
\hline $\mathrm{C}(20)-\mathrm{C}(1)-\mathrm{N}(1)$ & $127.4(4)$ \\
\hline$C(20)-C(1)-C(2)$ & $125.5(5)$ \\
\hline $\mathrm{N}(1)-\mathrm{C}(1)-\mathrm{C}(2)$ & $107.0(4)$ \\
\hline$C(3)-C(2)-C(1)$ & $109.3(5)$ \\
\hline $\mathrm{C}(2)-\mathrm{C}(3)-\mathrm{C}(4)$ & $108.0(4)$ \\
\hline $\mathrm{N}(1)-\mathrm{C}(4)-\mathrm{C}(5)$ & $125.5(4)$ \\
\hline $\mathrm{N}(1)-\mathrm{C}(4)-\mathrm{C}(3)$ & $108.3(4)$ \\
\hline$C(5)-C(4)-C(3)$ & $125.4(5)$ \\
\hline$C(6)-C(5)-C(4)$ & $122.9(5)$ \\
\hline$C(6)-C(5)-C(21)$ & $117.9(4)$ \\
\hline$C(4)-C(5)-C(21)$ & $118.0(4)$ \\
\hline $\mathrm{C}(5)-\mathrm{C}(6)-\mathrm{N}(2)$ & $125.1(4)$ \\
\hline
\end{tabular}




\begin{tabular}{|c|c|}
\hline$C(5)-C(6)-C(7)$ & $126.1(4)$ \\
\hline $\mathrm{N}(2)-\mathrm{C}(6)-\mathrm{C}(7)$ & $108.4(4)$ \\
\hline$C(8)-C(7)-C(6)$ & $108.0(4)$ \\
\hline$C(7)-C(8)-C(9)$ & $109.2(5)$ \\
\hline $\mathrm{C}(10)-\mathrm{C}(9)-\mathrm{N}(2)$ & $129.4(5)$ \\
\hline$C(10)-C(9)-C(8)$ & $124.1(5)$ \\
\hline $\mathrm{N}(2)-\mathrm{C}(9)-\mathrm{C}(8)$ & $106.5(4)$ \\
\hline $\mathrm{C}(9)-\mathrm{C}(10)-\mathrm{C}(11)$ & $127.9(5)$ \\
\hline $\mathrm{C}(9)-\mathrm{C}(10)-\mathrm{C}(27)$ & $115.8(4)$ \\
\hline $\mathrm{C}(11)-\mathrm{C}(10)-\mathrm{C}(27)$ & $116.3(4)$ \\
\hline $\mathrm{N}(3)-\mathrm{C}(11)-\mathrm{C}(10)$ & $126.2(5)$ \\
\hline $\mathrm{N}(3)-\mathrm{C}(11)-\mathrm{C}(12)$ & $108.8(4)$ \\
\hline $\mathrm{C}(10)-\mathrm{C}(11)-\mathrm{C}(12)$ & $124.9(5)$ \\
\hline$C(13)-C(12)-C(11)$ & $107.8(5)$ \\
\hline $\mathrm{C}(12)-\mathrm{C}(13)-\mathrm{C}(14)$ & $106.9(5)$ \\
\hline $\mathrm{N}(3)-\mathrm{C}(14)-\mathrm{C}(15)$ & $124.3(5)$ \\
\hline $\mathrm{N}(3)-\mathrm{C}(14)-\mathrm{C}(13)$ & $109.4(4)$ \\
\hline $\mathrm{C}(15)-\mathrm{C}(14)-\mathrm{C}(13)$ & $126.1(5)$ \\
\hline$C(16)-C(15)-C(14)$ & $123.4(4)$ \\
\hline$C(16)-C(15)-C(33)$ & $118.3(4)$ \\
\hline $\mathrm{C}(14)-\mathrm{C}(15)-\mathrm{C}(33)$ & $118.1(5)$ \\
\hline $\mathrm{N}(4)-\mathrm{C}(16)-\mathrm{C}(15)$ & $125.9(5)$ \\
\hline $\mathrm{N}(4)-\mathrm{C}(16)-\mathrm{C}(17)$ & $108.7(4)$ \\
\hline $\mathrm{C}(15)-\mathrm{C}(16)-\mathrm{C}(17)$ & $125.2(4)$ \\
\hline $\mathrm{C}(18)-\mathrm{C}(17)-\mathrm{C}(16)$ & $108.1(4)$ \\
\hline $\mathrm{C}(17)-\mathrm{C}(18)-\mathrm{C}(19)$ & $106.9(5)$ \\
\hline $\mathrm{N}(4)-\mathrm{C}(19)-\mathrm{C}(20)$ & $127.7(4)$ \\
\hline $\mathrm{N}(4)-\mathrm{C}(19)-\mathrm{C}(18)$ & $108.8(4)$ \\
\hline $\mathrm{C}(20)-\mathrm{C}(19)-\mathrm{C}(18)$ & $123.6(5)$ \\
\hline$C(19)-C(20)-C(1)$ & $126.9(5)$ \\
\hline
\end{tabular}




\begin{tabular}{|c|c|}
\hline $\mathrm{C}(19)-\mathrm{C}(20)-\mathrm{C}(39)$ & $116.1(4)$ \\
\hline$C(1)-C(20)-C(39)$ & $116.8(4)$ \\
\hline$C(22)-C(21)-C(26)$ & $118.4(5)$ \\
\hline$C(22)-C(21)-C(5)$ & $121.0(5)$ \\
\hline$C(26)-C(21)-C(5)$ & $120.6(5)$ \\
\hline$C(21)-C(22)-C(23)$ & $120.2(5)$ \\
\hline $\mathrm{C}(24)-\mathrm{C}(23)-\mathrm{C}(22)$ & $120.7(6)$ \\
\hline$C(23)-C(24)-C(25)$ & $120.2(5)$ \\
\hline$C(26)-C(25)-C(24)$ & $119.5(5)$ \\
\hline$C(25)-C(26)-C(21)$ & $121.0(5)$ \\
\hline $\mathrm{C}(28)-\mathrm{C}(27)-\mathrm{C}(32)$ & $118.0(5)$ \\
\hline $\mathrm{C}(28)-\mathrm{C}(27)-\mathrm{C}(10)$ & $120.8(5)$ \\
\hline $\mathrm{C}(32)-\mathrm{C}(27)-\mathrm{C}(10)$ & $121.2(5)$ \\
\hline$C(29)-C(28)-C(27)$ & $120.6(5)$ \\
\hline$C(28)-C(29)-C(30)$ & $120.9(5)$ \\
\hline $\mathrm{C}(31)-\mathrm{C}(30)-\mathrm{C}(29)$ & $119.0(5)$ \\
\hline $\mathrm{C}(30)-\mathrm{C}(31)-\mathrm{C}(32)$ & $121.2(6)$ \\
\hline $\mathrm{C}(31)-\mathrm{C}(32)-\mathrm{C}(27)$ & $120.3(5)$ \\
\hline $\mathrm{C}(34)-\mathrm{C}(33)-\mathrm{C}(38)$ & $116.9(5)$ \\
\hline $\mathrm{C}(34)-\mathrm{C}(33)-\mathrm{C}(15)$ & $124.1(5)$ \\
\hline $\mathrm{C}(38)-\mathrm{C}(33)-\mathrm{C}(15)$ & $119.0(5)$ \\
\hline $\mathrm{C}(33)-\mathrm{C}(34)-\mathrm{C}(35)$ & $121.7(6)$ \\
\hline $\mathrm{C}(36)-\mathrm{C}(35)-\mathrm{C}(34)$ & $121.0(6)$ \\
\hline $\mathrm{C}(35)-\mathrm{C}(36)-\mathrm{C}(37)$ & $119.4(6)$ \\
\hline $\mathrm{C}(36)-\mathrm{C}(37)-\mathrm{C}(38)$ & $119.8(7)$ \\
\hline $\mathrm{C}(37)-\mathrm{C}(38)-\mathrm{C}(33)$ & $120.9(6)$ \\
\hline $\mathrm{C}(44)-\mathrm{C}(39)-\mathrm{C}(40)$ & $118.4(5)$ \\
\hline $\mathrm{C}(44)-\mathrm{C}(39)-\mathrm{C}(20)$ & $123.1(5)$ \\
\hline $\mathrm{C}(40)-\mathrm{C}(39)-\mathrm{C}(20)$ & $118.5(5)$ \\
\hline $\mathrm{C}(41)-\mathrm{C}(40)-\mathrm{C}(39)$ & $120.4(5)$ \\
\hline
\end{tabular}




\begin{tabular}{|c|c|}
\hline $\mathrm{C}(40)-\mathrm{C}(41)-\mathrm{C}(42)$ & $119.8(5)$ \\
\hline $\mathrm{C}(43)-\mathrm{C}(42)-\mathrm{C}(41)$ & $120.1(5)$ \\
\hline $\mathrm{C}(42)-\mathrm{C}(43)-\mathrm{C}(44)$ & $120.0(6)$ \\
\hline C(39)-C(44)-C(43) & $121.3(5)$ \\
\hline $\mathrm{C}(46)-\mathrm{C}(45)-\mathrm{N}(1)$ & $121.1(4)$ \\
\hline $\mathrm{C}(46)-\mathrm{C}(45)-\mathrm{C}(47)$ & $122.9(4)$ \\
\hline $\mathrm{N}(1)-\mathrm{C}(45)-\mathrm{C}(47)$ & $115.0(4)$ \\
\hline $\mathrm{C}(45)-\mathrm{C}(46)-\mathrm{N}(2)$ & $120.2(4)$ \\
\hline $\mathrm{C}(45)-\mathrm{C}(46)-\mathrm{C}(53)$ & $125.9(5)$ \\
\hline$N(2)-C(46)-C(53)$ & $112.3(4)$ \\
\hline $\mathrm{C}(48)-\mathrm{C}(47)-\mathrm{C}(52)$ & $119.3(5)$ \\
\hline $\mathrm{C}(48)-\mathrm{C}(47)-\mathrm{C}(45)$ & $124.0(5)$ \\
\hline $\mathrm{C}(52)-\mathrm{C}(47)-\mathrm{C}(45)$ & $116.6(5)$ \\
\hline $\mathrm{C}(47)-\mathrm{C}(48)-\mathrm{C}(49)$ & $120.5(6)$ \\
\hline $\mathrm{C}(48)-\mathrm{C}(49)-\mathrm{C}(50)$ & $120.3(6)$ \\
\hline $\mathrm{C}(50)-\mathrm{C}(51)-\mathrm{C}(52)$ & $120.5(4)$ \\
\hline $\mathrm{C}(51)-\mathrm{C}(50)-\mathrm{C}(49)$ & $119.5(4)$ \\
\hline $\mathrm{C}(51)-\mathrm{C}(52)-\mathrm{C}(47)$ & $119.9(6)$ \\
\hline $\mathrm{C}(58)-\mathrm{C}(53)-\mathrm{C}(54)$ & $118.7(5)$ \\
\hline $\mathrm{C}(58)-\mathrm{C}(53)-\mathrm{C}(46)$ & $124.0(4)$ \\
\hline $\mathrm{C}(54)-\mathrm{C}(53)-\mathrm{C}(46)$ & $117.1(4)$ \\
\hline$C(55)-C(54)-C(53)$ & $120.4(5)$ \\
\hline $\mathrm{C}(56)-\mathrm{C}(55)-\mathrm{C}(54)$ & $120.9(5)$ \\
\hline$C(55)-C(56)-C(57)$ & $119.7(5)$ \\
\hline $\mathrm{C}(56)-\mathrm{C}(57)-\mathrm{C}(58)$ & $120.4(5)$ \\
\hline $\mathrm{C}(53)-\mathrm{C}(58)-\mathrm{C}(57)$ & $119.9(5)$ \\
\hline $\mathrm{O}(1 \mathrm{~A})-\mathrm{C}(59)-\mathrm{Pd}(1)$ & $169.6(7)$ \\
\hline $\mathrm{O}(1 \mathrm{~B})-\mathrm{C}(60)-\mathrm{Pd}(1)$ & $169.7(10)$ \\
\hline $\mathrm{O}(6)-\mathrm{C}(61)-\mathrm{C}(62)$ & $115.9(13)$ \\
\hline $\mathrm{O}(6)-\mathrm{C}(63)-\mathrm{C}(64)$ & $122.2(13)$ \\
\hline
\end{tabular}




\begin{tabular}{|c|c|}
\hline $\mathrm{O}(4)-\mathrm{Cl}(1)-\mathrm{O}(5)$ & $105.9(10)$ \\
\hline $\mathrm{O}(4)-\mathrm{Cl}(1)-\mathrm{O}(2)$ & $110.8(7)$ \\
\hline $\mathrm{O}(5)-\mathrm{Cl}(1)-\mathrm{O}(2)$ & $108.7(5)$ \\
\hline $\mathrm{O}(4)-\mathrm{Cl}(1)-\mathrm{O}(3)$ & $105.9(5)$ \\
\hline $\mathrm{O}(5)-\mathrm{Cl}(1)-\mathrm{O}(3)$ & $112.4(6)$ \\
\hline $\mathrm{O}(2)-\mathrm{Cl}(1)-\mathrm{O}(3)$ & $112.9(3)$ \\
\hline $\mathrm{C}(4)-\mathrm{N}(1)-\mathrm{C}(1)$ & $107.3(4)$ \\
\hline $\mathrm{C}(4)-\mathrm{N}(1)-\mathrm{C}(45)$ & 119.7(4) \\
\hline $\mathrm{C}(1)-\mathrm{N}(1)-\mathrm{C}(45)$ & $125.1(4)$ \\
\hline $\mathrm{C}(6)-\mathrm{N}(2)-\mathrm{C}(9)$ & $107.9(4)$ \\
\hline $\mathrm{C}(6)-\mathrm{N}(2)-\mathrm{C}(46)$ & 119.7(4) \\
\hline $\mathrm{C}(9)-\mathrm{N}(2)-\mathrm{C}(46)$ & $122.6(4)$ \\
\hline $\mathrm{C}(14)-\mathrm{N}(3)-\mathrm{C}(11)$ & $107.0(4)$ \\
\hline $\mathrm{C}(14)-\mathrm{N}(3)-\mathrm{Pd}(1)$ & $116.9(3)$ \\
\hline $\mathrm{C}(11)-\mathrm{N}(3)-\mathrm{Pd}(1)$ & $130.7(3)$ \\
\hline $\mathrm{C}(19)-\mathrm{N}(4)-\mathrm{C}(16)$ & $107.6(4)$ \\
\hline$C(19)-N(4)-\operatorname{Pd}(1)$ & 131.1(3) \\
\hline $\mathrm{C}(16)-\mathrm{N}(4)-\mathrm{Pd}(1)$ & 116.2(3) \\
\hline $\mathrm{C}(63)-\mathrm{O}(6)-\mathrm{C}(61)$ & $124.0(12)$ \\
\hline$C(59)-P d(1)-C(60)$ & $85.0(3)$ \\
\hline $\mathrm{C}(59)-\mathrm{Pd}(1)-\mathrm{N}(3)$ & $176.8(2)$ \\
\hline $\mathrm{C}(60)-\mathrm{Pd}(1)-\mathrm{N}(3)$ & $94.2(2)$ \\
\hline $\mathrm{C}(59)-\mathrm{Pd}(1)-\mathrm{N}(4)$ & $96.4(2)$ \\
\hline $\mathrm{C}(60)-\mathrm{Pd}(1)-\mathrm{N}(4)$ & $174.2(2)$ \\
\hline $\mathrm{N}(3)-\mathrm{Pd}(1)-\mathrm{N}(4)$ & $84.13(16)$ \\
\hline
\end{tabular}

Symmetry transformations used to generate equivalent atoms: 
Table 10. Anisotropic displacement parameters $\left(\AA^{2} \times 10^{3}\right)$ for (5). The anisotropic displacement factor exponent takes the form: $-2 p 2\left[h 2 a * 2 U_{11}+\ldots+2 h k a^{*} b^{*} U_{12}\right]$

\begin{tabular}{|c|c|c|c|c|c|c|}
\hline & $\mathrm{U}_{11}$ & $\mathrm{U}_{22}$ & $\mathrm{U}_{33}$ & $\mathrm{U}_{23}$ & $\mathrm{U}_{13}$ & $\mathrm{U}_{12}$ \\
\hline $\mathrm{C}(1)$ & $21(3)$ & $25(3)$ & $20(3)$ & $1(2)$ & $5(2)$ & $2(2)$ \\
\hline$C(2)$ & $19(3)$ & $33(3)$ & $34(3)$ & $-3(2)$ & $5(2)$ & $-5(2)$ \\
\hline $\mathrm{C}(3)$ & $18(3)$ & $35(3)$ & $34(3)$ & $-4(2)$ & $12(2)$ & $-1(2)$ \\
\hline$C(4)$ & $21(3)$ & $29(3)$ & $21(3)$ & $5(2)$ & $4(2)$ & $-1(2)$ \\
\hline$C(5)$ & $22(3)$ & $27(3)$ & $20(3)$ & $3(2)$ & $5(2)$ & $3(2)$ \\
\hline$C(6)$ & $21(3)$ & $27(3)$ & $22(3)$ & $4(2)$ & $7(2)$ & $3(2)$ \\
\hline$C(7)$ & $22(3)$ & $26(3)$ & $33(3)$ & $1(2)$ & $7(2)$ & $2(2)$ \\
\hline $\mathrm{C}(8)$ & $23(3)$ & $22(3)$ & $33(3)$ & $5(2)$ & $7(2)$ & $-1(2)$ \\
\hline $\mathrm{C}(9)$ & $22(3)$ & $23(3)$ & $22(3)$ & $11(2)$ & $1(2)$ & $-3(2)$ \\
\hline $\mathrm{C}(10)$ & $22(3)$ & $29(3)$ & $23(3)$ & $10(2)$ & $2(2)$ & $-1(2)$ \\
\hline $\mathrm{C}(11)$ & $21(3)$ & $28(3)$ & $26(3)$ & $8(2)$ & $7(2)$ & $-1(2)$ \\
\hline $\mathrm{C}(12)$ & $27(3)$ & $31(3)$ & $41(3)$ & $6(3)$ & $8(2)$ & $-5(2)$ \\
\hline $\mathrm{C}(13)$ & $23(3)$ & $35(3)$ & $41(3)$ & $7(3)$ & $16(2)$ & $7(2)$ \\
\hline $\mathrm{C}(14)$ & $21(3)$ & $32(3)$ & $27(3)$ & $12(2)$ & $6(2)$ & $0(2)$ \\
\hline $\mathrm{C}(15)$ & $18(3)$ & $33(3)$ & $20(3)$ & $10(2)$ & $5(2)$ & $6(2)$ \\
\hline$C(16)$ & $19(3)$ & $32(3)$ & $18(3)$ & $6(2)$ & $4(2)$ & $7(2)$ \\
\hline $\mathrm{C}(17)$ & $23(3)$ & $30(3)$ & $27(3)$ & $3(2)$ & $8(2)$ & $9(2)$ \\
\hline $\mathrm{C}(18)$ & $24(3)$ & $23(3)$ & $29(3)$ & $5(2)$ & $7(2)$ & $5(2)$ \\
\hline $\mathrm{C}(19)$ & $19(2)$ & $27(3)$ & $20(3)$ & $5(2)$ & $2(2)$ & $2(2)$ \\
\hline $\mathrm{C}(20)$ & $24(3)$ & $23(3)$ & $19(3)$ & $3(2)$ & $3(2)$ & $1(2)$ \\
\hline $\mathrm{C}(21)$ & $16(2)$ & $23(3)$ & $25(3)$ & $0(2)$ & $4(2)$ & $0(2)$ \\
\hline $\mathrm{C}(22)$ & $22(3)$ & $37(3)$ & $30(3)$ & $7(2)$ & $6(2)$ & $6(2)$ \\
\hline $\mathrm{C}(23)$ & $32(3)$ & $45(3)$ & $27(3)$ & $4(3)$ & $12(2)$ & $6(3)$ \\
\hline $\mathrm{C}(24)$ & $24(3)$ & $32(3)$ & $35(3)$ & $-5(3)$ & $10(2)$ & $6(2)$ \\
\hline$C(25)$ & $18(3)$ & $36(3)$ & $43(3)$ & $10(3)$ & $4(2)$ & $9(2)$ \\
\hline$C(26)$ & $30(3)$ & $52(4)$ & $32(3)$ & $11(3)$ & $7(2)$ & $8(3)$ \\
\hline
\end{tabular}




$\begin{array}{lllllll}\mathrm{C}(27) & 20(3) & 24(3) & 34(3) & 7(2) & 4(2) & 1(2) \\ \mathrm{C}(28) & 24(3) & 38(3) & 33(3) & 14(3) & 10(2) & 3(2) \\ \mathrm{C}(29) & 33(3) & 38(3) & 35(3) & 3(3) & 6(2) & -7(3) \\ \mathrm{C}(30) & 35(3) & 29(3) & 46(4) & 5(3) & 5(3) & -9(3) \\ \mathrm{C}(31) & 33(3) & 36(3) & 45(4) & 21(3) & 8(3) & -1(3) \\ \mathrm{C}(32) & 27(3) & 31(3) & 31(3) & 11(2) & 4(2) & -3(2) \\ \mathrm{C}(33) & 20(3) & 38(3) & 29(3) & 12(2) & 13(2) & 10(2) \\ \mathrm{C}(34) & 39(3) & 66(4) & 25(3) & 5(3) & 5(3) & 23(3) \\ \mathrm{C}(35) & 29(3) & 55(4) & 47(4) & 7(3) & 3(3) & 22(3) \\ \mathrm{C}(36) & 45(4) & 78(5) & 52(4) & 26(4) & 31(3) & 32(4) \\ \mathrm{C}(37) & 121(8) & 253(14) & 51(5) & 81(7) & 60(5) & 143(10) \\ \mathrm{C}(38) & 82(6) & 210(11) & 41(4) & 55(6) & 27(4) & 112(7) \\ \mathrm{C}(39) & 20(3) & 25(3) & 29(3) & 2(2) & 4(2) & 4(2) \\ \mathrm{C}(40) & 22(3) & 33(3) & 26(3) & 2(2) & 8(2) & 10(2) \\ \mathrm{C}(41) & 27(3) & 31(3) & 30(3) & -3(2) & -1(2) & 7(2) \\ \mathrm{C}(42) & 22(3) & 25(3) & 53(4) & -4(3) & -3(3) & 2(2) \\ \mathrm{C}(43) & 25(3) & 32(3) & 51(4) & 12(3) & 12(3) & 3(2) \\ \mathrm{C}(44) & 31(3) & 26(3) & 31(3) & 1(2) & 9(2) & -2(2) \\ \mathrm{C}(45) & 20(2) & 24(3) & 15(2) & 2(2) & 3(2) & 1(2) \\ \mathrm{C}(46) & 19(2) & 23(3) & 20(3) & 3(2) & 5(2) & 3(2) \\ \mathrm{C}(47) & 26(3) & 24(3) & 21(3) & 3(2) & -3(2) & -4(2) \\ \mathrm{C}(48) & 31(3) & 33(3) & 27(3) & 8(2) & -3(2) & 1(2) \\ \mathrm{C}(49) & 53(4) & 30(3) & 60(5) & 12(3) & -12(3) & 7(3) \\ \mathrm{C}(51) & 70(5) & 55(5) & 55(5) & 33(4) & 7(4) & -17(4) \\ \mathrm{C}(50) & 80(6) & 32(4) & 66(5) & 31(3) & -3(4) & -2(4) \\ \mathrm{C}(52) & 37(3) & 39(3) & 32(3) & 15(3) & 6(2) & -7(3) \\ \mathrm{C}(5) & 20(2) & 22(3) & 21(3) & 9(2) & 4(2) & -2(2) \\ \mathrm{C}(3) & 33(3) & 23(3) & 8(2) & 2(2) & 0(2) \\ & 39(3) & 40(3) & 36(3) & 14(3) & -8(2) & -7(3)\end{array}$




\begin{tabular}{|c|c|c|c|c|c|c|}
\hline$C(57)$ & $26(3)$ & $47(4)$ & $42(4)$ & $19(3)$ & $3(3)$ & $7(3)$ \\
\hline$C(58)$ & $23(3)$ & $31(3)$ & $28(3)$ & $6(2)$ & $4(2)$ & $3(2)$ \\
\hline$C(59)$ & $41(4)$ & $57(4)$ & $38(4)$ & $30(3)$ & $21(3)$ & $20(3)$ \\
\hline$C(60)$ & $36(4)$ & $55(4)$ & $29(3)$ & $11(3)$ & $7(3)$ & $19(3)$ \\
\hline$C(61)$ & $174(15)$ & $252(19)$ & 131(12) & $67(12)$ & $49(11)$ & $148(15)$ \\
\hline$C(62)$ & $256(19)$ & $170(13)$ & $124(11)$ & $-9(9)$ & $132(12)$ & $-102(13)$ \\
\hline$C(63)$ & $330(20)$ & $510(40)$ & $46(7)$ & $-3(12)$ & $-9(11)$ & $360(30)$ \\
\hline$C(64)$ & 281(19) & $62(6)$ & $73(7)$ & $7(5)$ & $52(10)$ & $-16(9)$ \\
\hline $\mathrm{Cl}(1)$ & $96(1)$ & $34(1)$ & $45(1)$ & $-8(1)$ & $46(1)$ & $-18(1)$ \\
\hline $\mathrm{N}(1)$ & $15(2)$ & $24(2)$ & $17(2)$ & $1(2)$ & $8(2)$ & $2(2)$ \\
\hline $\mathrm{N}(2)$ & $18(2)$ & $20(2)$ & $22(2)$ & $6(2)$ & $5(2)$ & $1(2)$ \\
\hline $\mathrm{N}(3)$ & $20(2)$ & $30(2)$ & $22(2)$ & $9(2)$ & $7(2)$ & $3(2)$ \\
\hline $\mathrm{N}(4)$ & $17(2)$ & $24(2)$ & $21(2)$ & $5(2)$ & $5(2)$ & $4(2)$ \\
\hline $\mathrm{O}(2)$ & 101(4) & $63(3)$ & $44(3)$ & $8(2)$ & $32(3)$ & $-18(3)$ \\
\hline $\mathrm{O}(3)$ & $82(4)$ & $51(3)$ & $68(4)$ & $14(3)$ & $3(3)$ & $-13(3)$ \\
\hline $\mathrm{O}(4)$ & $376(18)$ & $166(9)$ & $510(20)$ & $195(12)$ & 413(19) & $182(11)$ \\
\hline $\mathrm{O}(5)$ & $500(20)$ & $204(10)$ & $37(4)$ & $16(5)$ & $-34(7)$ & $-268(13)$ \\
\hline $\mathrm{O}(6)$ & $173(8)$ & $97(5)$ & $79(5)$ & $28(4)$ & $46(5)$ & $-6(5)$ \\
\hline $\mathrm{O}(1 \mathrm{~A})$ & $18(4)$ & $74(6)$ & $69(6)$ & $40(5)$ & $-12(3)$ & $0(4)$ \\
\hline $\mathrm{O}(1 \mathrm{~B})$ & $73(9)$ & $43(8)$ & $74(9)$ & $22(7)$ & $-7(7)$ & $19(7)$ \\
\hline $\operatorname{Pd}(1)$ & $19(1)$ & $29(1)$ & $21(1)$ & $7(1)$ & $6(1)$ & $6(1)$ \\
\hline
\end{tabular}

Table 11. Hydrogen coordinates $\left(x 10^{4}\right)$ and isotropic displacement parameters $\left(\AA^{2} \times 10^{3}\right)$ for (5).

\begin{tabular}{lllll}
\hline & $\mathrm{x}$ & $\mathrm{y}$ & $\mathrm{z}$ & $\mathrm{U}(\mathrm{eq})$ \\
$\mathrm{H}(2)$ & 1745 & 8000 & 6689 & 38
\end{tabular}




\begin{tabular}{|c|c|c|c|}
\hline $\mathrm{H}(3)$ & 1565 & 9600 & 7799 \\
\hline $\mathrm{H}(7)$ & 4052 & 13042 & 8929 \\
\hline $\mathrm{H}(8)$ & 5803 & 13550 & 8562 \\
\hline $\mathrm{H}(12)$ & 9214 & 11649 & 7323 \\
\hline $\mathrm{H}(13)$ & 9406 & 10015 & 6245 \\
\hline $\mathrm{H}(17)$ & 6930 & 6494 & 5195 \\
\hline $\mathrm{H}(18)$ & 5164 & 5966 & 5498 \\
\hline $\mathrm{H}(22)$ & 2841 & 11000 & 9850 \\
\hline $\mathrm{H}(23)$ & 1424 & 11863 & 10491 \\
\hline $\mathrm{H}(24)$ & 304 & 12823 & 9856 \\
\hline $\mathrm{H}(25)$ & 574 & 12937 & 8554 \\
\hline $\mathrm{H}(26)$ & 1984 & 12081 & 7904 \\
\hline $\mathrm{H}(28)$ & 7873 & 13141 & 9348 \\
\hline $\mathrm{H}(29)$ & 8849 & 14718 & 9843 \\
\hline $\mathrm{H}(30)$ & 9386 & 15512 & 8918 \\
\hline $\mathrm{H}(31)$ & 8924 & 14716 & 7489 \\
\hline $\mathrm{H}(32)$ & 7932 & 13148 & 6973 \\
\hline $\mathrm{H}(34)$ & 9463 & 7910 & 6131 \\
\hline $\mathrm{H}(35)$ & 10716 & 7138 & 5287 \\
\hline $\mathrm{H}(36)$ & 10415 & 6944 & 3891 \\
\hline $\mathrm{H}(37)$ & 8693 & 7260 & 3278 \\
\hline $\mathrm{H}(38)$ & 7452 & 8119 & 4124 \\
\hline $\mathrm{H}(40)$ & 3386 & 6653 & 4642 \\
\hline $\mathrm{H}(41)$ & 2177 & 5251 & 3869 \\
\hline $\mathrm{H}(42)$ & 1086 & 4471 & 4554 \\
\hline $\mathrm{H}(43)$ & 1287 & 5023 & 6006 \\
\hline $\mathrm{H}(44)$ & 2528 & 6393 & 6781 \\
\hline $\mathrm{H}(48)$ & 6841 & 8011 & 7552 \\
\hline $\mathrm{H}(49)$ & 7259 & 6597 & 7984 \\
\hline $\mathrm{H}(51)$ & 4623 & 6976 & 9190 \\
\hline
\end{tabular}




\begin{tabular}{|c|c|c|c|c|}
\hline $\mathrm{H}(50)$ & 6174 & 6102 & 8840 & 69 \\
\hline $\mathrm{H}(52)$ & 4178 & 8378 & 8739 & 42 \\
\hline $\mathrm{H}(54)$ & 6155 & 11293 & 9751 & 35 \\
\hline $\mathrm{H}(55)$ & 7737 & 11722 & 10733 & 42 \\
\hline $\mathrm{H}(56)$ & 9418 & 11142 & 10384 & 44 \\
\hline $\mathrm{H}(57)$ & 9511 & 10047 & 9045 & 45 \\
\hline $\mathrm{H}(58)$ & 7919 & 9564 & 8046 & 33 \\
\hline $\mathrm{H}(61 \mathrm{~A})$ & 2201 & 4421 & 7673 & 216 \\
\hline $\mathrm{H}(61 \mathrm{~B})$ & 2507 & 5581 & 7796 & 216 \\
\hline $\mathrm{H}(62 \mathrm{~A})$ & 3269 & 5143 & 9151 & 280 \\
\hline $\mathrm{H}(62 \mathrm{~B})$ & 1995 & 4820 & 8884 & 280 \\
\hline $\mathrm{H}(62 \mathrm{C})$ & 2421 & 5961 & 9030 & 280 \\
\hline $\mathrm{H}(63 \mathrm{~A})$ & 3645 & 4898 & 6789 & 380 \\
\hline $\mathrm{H}(63 \mathrm{~B})$ & 3631 & 3770 & 6821 & 380 \\
\hline $\mathrm{H}(64 \mathrm{~A})$ & 5667 & 4683 & 7445 & 209 \\
\hline $\mathrm{H}(64 \mathrm{~B})$ & 5280 & 4743 & 6534 & 209 \\
\hline $\mathrm{H}(64 \mathrm{C})$ & 5322 & 3669 & 6692 & 209 \\
\hline $\mathrm{H}(59 \mathrm{~A})$ & 3386 & 10264 & 5575 & 28 \\
\hline $\mathrm{H}(59 \mathrm{~B})$ & 3679 & 9164 & 5049 & 28 \\
\hline $\mathrm{H}(59 \mathrm{C})$ & 3294 & 9332 & 5927 & 28 \\
\hline $\mathrm{H}(60 \mathrm{~A})$ & 4578 & 11700 & 6549 & 28 \\
\hline $\mathrm{H}(60 \mathrm{~B})$ & 5742 & 11749 & 6261 & 28 \\
\hline $\mathrm{H}(60 \mathrm{C})$ & 4673 & 11348 & 5586 & 28 \\
\hline
\end{tabular}

Table 12. Least-squares planes ( $\mathrm{x}, \mathrm{y}, \mathrm{z}$ in crystal coordinates) and deviations from them (* indicates atom used to define plane) for (5). 
PLANE 1

$5.9555(0.0139) \mathrm{x}-8.1884(0.0152) \mathrm{y}+12.0160(0.0149) \mathrm{z}=3.7855(0.0209)$

* $0.0034(0.0020) \mathrm{N} 1$

* $-0.0034(0.0020) \mathrm{N} 2$

* $0.0034(0.0019) \mathrm{N} 3$

* $-0.0034(0.0020) \mathrm{N} 4$

$-1.3776(0.0023)$ Pd1

Rms deviation of fitted atoms $=0.0034$

\section{PLANE 2}

$-4.7284(0.0215) x-1.7802(0.0263) y+16.0975(0.0095) z=5.6641(0.0279)$

Angle to previous plane (with approximate esd) $=61.85(0.11)$

* $0.0000(0.0000) \mathrm{N} 3$

* $\quad 0.0000(0.0000) \mathrm{N} 4$

* $0.0000(0.0000) \mathrm{Pd} 1$

Rms deviation of fitted atoms $=0.0000$

PLANE 3

$4.4968(0.0112) \mathrm{x}-7.9443(0.0115) \mathrm{y}+13.6411(0.0097) \mathrm{z}=4.3163(0.0156)$

Angle to previous plane (with approximate esd) $=53.29(0.10)$

* $0.0338(0.0024)$ C5

* $-0.0341(0.0024) \mathrm{C} 10$

* $0.0344(0.0025) \mathrm{C} 15$

* $-0.0341(0.0025) \mathrm{C} 20$

$-1.4324(0.0025) \mathrm{Pd} 1$

Rms deviation of fitted atoms $=0.0341$

\section{PLANE 4}

$-5.0804(0.0181) x-1.2046(0.0235) y+15.8305(0.0096) z=5.8092(0.0243)$

Angle to previous plane (with approximate esd) $=56.13(0.09)$ 
* $\quad 0.0217(0.0025) \mathrm{N} 3$

* $-0.0215(0.0025) \mathrm{N} 4$

* $0.0227(0.0026)$ C59

* $-0.0229(0.0027)$ C60

$0.0768(0.0027) \operatorname{Pd} 1$

Rms deviation of fitted atoms $=0.0222$

\section{PLANE 5}

$0.5801(0.0332) \mathrm{x}-9.7359(0.0279) \mathrm{y}+14.6746(0.0241) \mathrm{z}=2.1479(0.0422)$

Angle to the PLANE 3 (with approximate esd) $=20.19(0.23$ )

* $0.0121(0.0030) \mathrm{N} 1$

* $-0.0038(0.0033) \mathrm{C} 1$

* $-0.0060(0.0037) \mathrm{C} 2$

* $0.0135(0.0037) \mathrm{C} 3$

* $-0.0158(0.0033) \mathrm{C} 4$

Rms deviation of fitted atoms $=0.0112$

PLANE 6

$4.0226(0.0301) \mathrm{x}-3.8628(0.0335) \mathrm{y}+14.9425(0.0206) \mathrm{z}=9.9094(0.0393)$

Angle to the PLANE 3 (with approximate esd) $=20.03(0.23$ )

* $0.0049(0.0028) \mathrm{N} 2$

* $-0.0080(0.0030) \mathrm{C} 6$

* $0.0082(0.0033) \mathrm{C} 7$

* $-0.0051(0.0032) \mathrm{C} 8$

* $0.0001(0.0029)$ C9

Rms deviation of fitted atoms $=0.0060$

\section{PLANE 7}

$1.6656(0.0329) x-9.0868(0.0302) y+14.7658(0.0240) z=1.7327(0.0490)$

Angle to the PLANE 3 (with approximate esd) $=14.28(0.26)$ 
* $-0.0082(0.0031) \mathrm{N} 3$

* $-0.0010(0.0033) \mathrm{C} 11$

* $0.0098(0.0037) \mathrm{C} 12$

* $-0.0146(0.0036) \mathrm{C} 13$

* $0.0140(0.0033) \mathrm{C} 14$

$-0.7818(0.0084) \operatorname{Pd} 1$

Rms deviation of fitted atoms $=0.0107$

PLANE 8

$3.8135(0.0307) \mathrm{x}-4.4188(0.0317) \mathrm{y}+15.0779(0.0200) \mathrm{z}=7.6120(0.0265)$

Angle to the PLANE 3 (with approximate esd) $=17.94(0.23)$

* $\quad 0.0036(0.0029) \mathrm{N} 4$

* $-0.0011(0.0029) \mathrm{C} 16$

* $\quad-0.0017$ (0.0032) $\mathrm{C} 17$

* $0.0037(0.0032) \mathrm{C} 18$

* $-0.0044(0.0029)$ C19

$-0.6993(0.0077)$ Pd1

Rms deviation of fitted atoms $=0.0032$ 
Figure 1. Ortep drawings with atom numbering scheme (50\% level thermal ellipsoids) of (4).

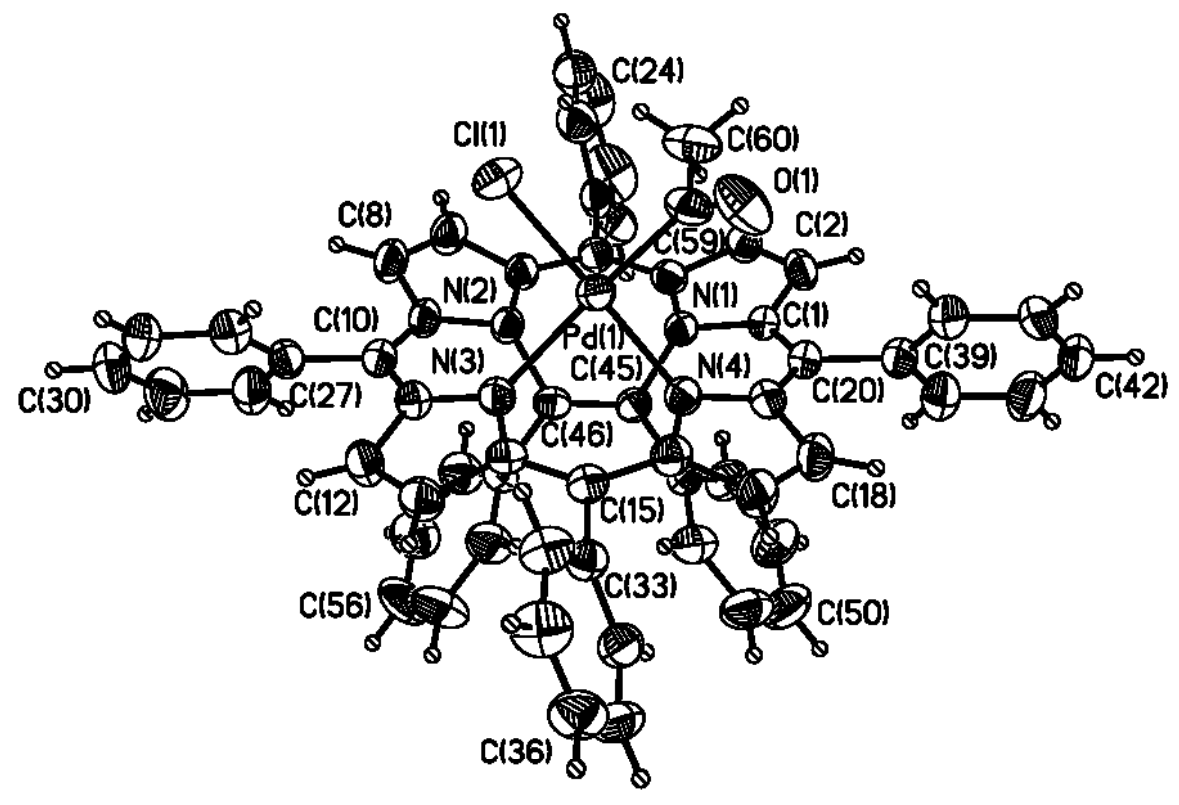


Figure 2. Ortep drawing with atom numbering scheme (50\% level thermal ellipsoids) of (5).

$\mathrm{X}$ ray structure (top view and side view) of (por) $\mathrm{Pd}-\left(\mathrm{CH}_{3}\right)(\mathrm{CO}) \mathrm{ClO}_{4}(\mathbf{5})$ with atom numbering scheme. Six phenyl groups (C21-26; C27-32; C33-38; C39-44; C47-52; C53-58) at C5, C10, C15, C20, C45, and C46 were omitted for clarity in the middle and bottom and the occupancy of the carbonyl oxygens is 0.59 for O1A and 0.41 for O1B.
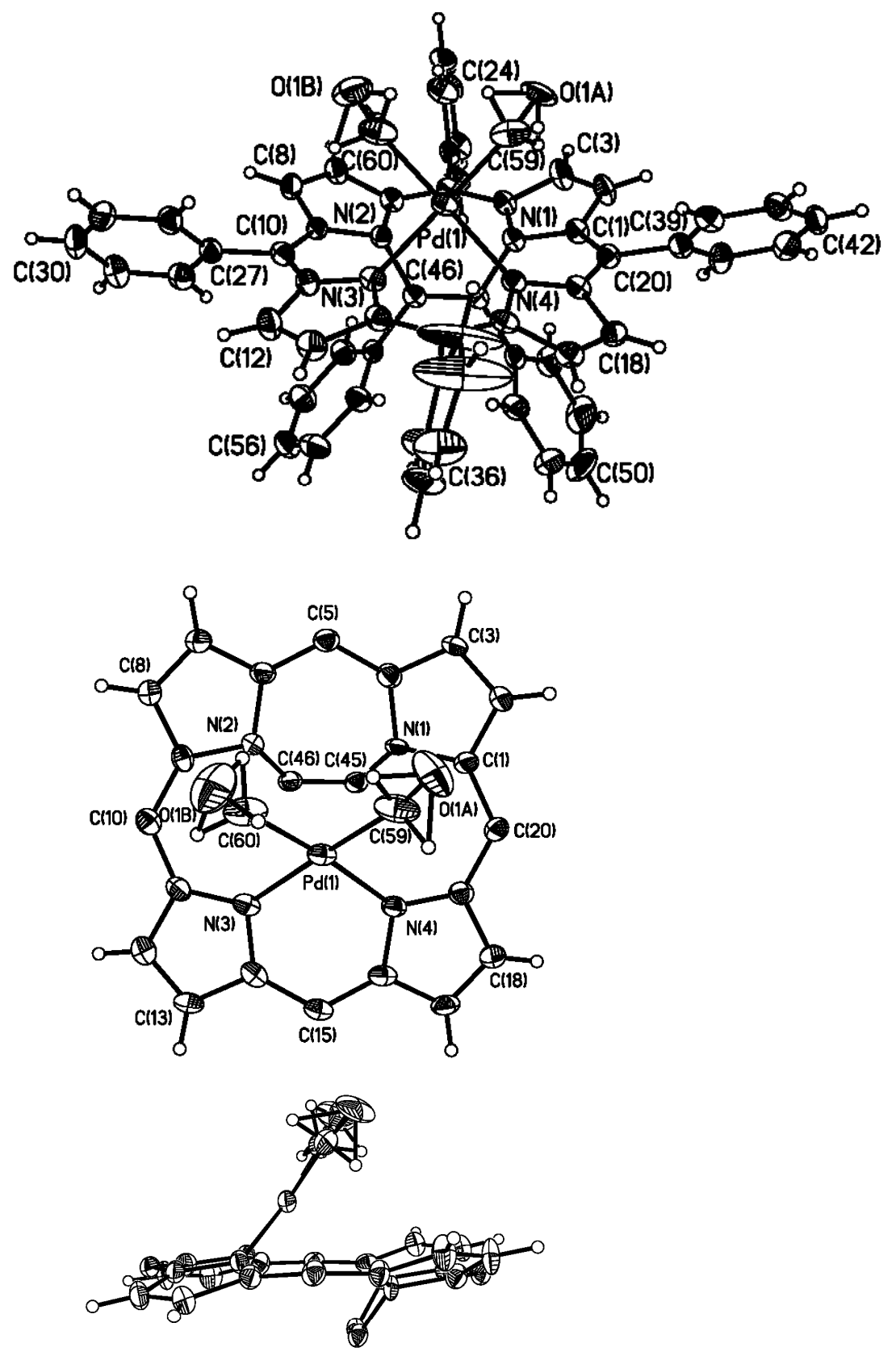